\title{
Bioanalysis
}

\section{White Paper on Recent Issues in Bioanalysis: focus on flow cytometry, gene therapy, cut points and key clarifications on BAV (Part 3 - LBA/cell-based assays: immunogenicity, biomarkers and PK assays)}

The $201812^{\text {th }}$ Workshop on Recent Issues in Bioanalysis took place in Philadelphia, PA, USA on April 9-13, 2018 with an attendance of over 900 representatives from pharmaceutical/biopharmaceutical companies, biotechnology companies, contract research organizations and regulatory agencies worldwide. WRIB was once again a 5-day full immersion in bioanalysis, biomarkers and immunogenicity. As usual, it was specifically designed to facilitate sharing, reviewing, discussing and agreeing on approaches to address the most current issues of interest including both small- and large-molecule bioanalysis involving LCMS, hybrid LBA/LCMS and LBA/cell-based assays approaches. This 2018 White Paper encompasses recommendations emerging from the extensive discussions held during the workshop and is aimed to provide the bioanalytical community with key information and practical solutions on topics and issues addressed, in an effort to enable advances in scientific excellence, improved quality and better regulatory compliance. Due to its length, the 2018 edition of this comprehensive White Paper has been divided into three parts for editorial reasons. This publication (Part 3) covers the recommendations for large molecule bioanalysis, biomarkers and immunogenicity using LBA and cell-based assays. Part 1 (LCMS for small molecules, peptides, oligonucleotides and small molecule biomarkers) and Part 2 (hybrid LBA/LCMS for biotherapeutics and regulatory agencies' inputs) are published in volume 10 of Bioanalysis, issues 22 and 23 (2018), respectively.

First draft submitted: 29 September 2018; Accepted for publication: 29 October 2018; Published online: 29 November 2018

Index

Introduction

Discussion topics

- Gene therapy

- Critical reagents

- Biomarker assay validation

- Flow cytometry

- ADA assays \& PK, PD, ADA data correlation

- Cut points

Discussions, consensus $\&$ conclusions

- Gene therapy

- Critical reagents

- Biomarker assay validation

- Flow cytometry

- ADA assays \& PK, PD, ADA data correlation

- Cut points

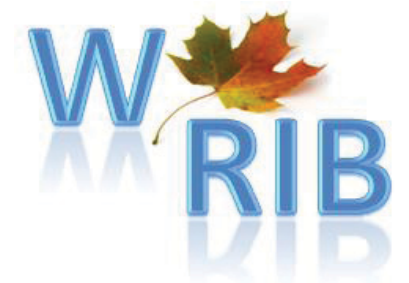

Lauren Stevenson ${ }^{1}$, Susan Richards², Renuka Pillutla ${ }^{3}$, Albert Torri $^{4}$, John Kamerud ${ }^{5}$, Devangi Mehta ${ }^{1}$, Stephen Keller $^{6}$, Shobha

Purushothama ${ }^{1}$, Boris Gorovits ${ }^{5}$, Virginia Litwin? Christopher Stebbins ${ }^{1}$, Joseph Marini $^{8}$, Chris Beaver ${ }^{9}$, Gizette Sperinde $^{10}$, Patricia Siguenza ${ }^{10}$, Roland F Staack ${ }^{11}$, Yongchang Qiu'12, Lakshmi Amaravadi ${ }^{13}$, Shashi Amur ${ }^{14}$, Catherine Aversa Fleener ${ }^{15}$, Daniel Baltrukonis $^{16}$, Ian Catlett ${ }^{3}$, Elana Cherry ${ }^{17}$, Shan Chung ${ }^{10}$, Isabelle Cludts ${ }^{18}$, Lorella Di Donato $^{7}$, Saloumeh Fischer ${ }^{10}$, Stephanie Fraser ${ }^{16}$, Fabio Garofolo*,19, Cherie Green ${ }^{10}$, George Gunn ${ }^{20}$, Sam Haidar ${ }^{14}$, Jonathan Haulenbeek ${ }^{3}$, Neil Henderson ${ }^{21}$, Shirley Hopper ${ }^{22}$, Akiko Ishii-Watabe ${ }^{23}$, Rafiq Islam ${ }^{24}$, Brian Janelsins ${ }^{14}$, Vibha Jawa ${ }^{25}$, Vellalore Kakkanaiah ${ }^{26}$, Szilard Kamondi ${ }^{27}$, Gerry Kolaitis ${ }^{3}$, Robert J Kubiak ${ }^{28}$, Seema Kumar $^{29}$, Pekka Kurki ${ }^{30}$, Meina Liang ${ }^{31}$, Patrick Liu ${ }^{32}$, Kimberly Maxfield ${ }^{14}$, Heather Myler $^{26}$, Nisha Palackal ${ }^{4}$, Rachel Palmer², João Pedras-Vasconcelos ${ }^{14}$, Steven Piccoli ${ }^{33}$, Paul Rhyne ${ }^{34}$, Yoshiro Saito $^{23}$, Natasha Savoie ${ }^{35}$, Eginhard Schick ${ }^{27}$, Becky Schweighardt ${ }^{36}$ \& Judy Shih ${ }^{37}$

*Author for correspondence:

f.garofolo@angelini.it

The remaining list of author names, affiliations and Regulatory Agencies Disclaimer can be found at the end of the article

newlands 
Recommendations

- Gene therapy

- Critical reagents

- Biomarker assay validation

- Flow cytometry

- ADA assays \& PK, PD, ADA data correlation

- Cut points

References

\section{Key terms}

Biomarker: A defined characteristic that is measured as an indicator of normal biological processes, pathogenic processes, or responses to an exposure or intervention, including therapeutic interventions. Molecular, histologic, radiographic, or physiologic characteristics are types of biomarkers. Categories of biomarkers include: susceptibility/risk biomarker, diagnostic biomarker, monitoring biomarker, prognostic biomarker, predictive biomarker, pharmacodynamic/response biomarker, and safety biomarker [43].

Biomarker qualification: A conclusion based on a formal regulatory process, that within the stated context of use, a medical product development tool can be relied upon to have a specific interpretation and application in medical product development and regulatory review [43].

Immunogenicity: The ability of a substance, including biotherapeutic substances, to provoke an immune response in vivo which results in an induction of anti-drug antibodies, via humoral immune response and/or cytotoxic T-cell induction via cellular immune response - among others. For the purposes of this article, immunogenicity is defined as induction of ADA and ADA assays are equivalent to immunogenicity assays.

Cut point: The minimum level of response in the assay that defines the sample as positive for ADA.

Context of use (COU): A statement that fully and clearly describes the way the medical product development tool is to be used and the medical product development-related purpose of the use [43].

Biomarker assay validation (BAV) \& analytical validation: In the context of this paper, biomarker assay validation is the same as analytical validation. Over the last several years, the US FDA as well as others in the scientific community have co-sponsored multiple workshops aimed at advancing the discussion around biomarker assay validation.

\section{Acronyms}

\begin{tabular}{|ll|}
\hline AAV: & Adeno-associated virus \\
\hline Abs: & Antibodies \\
\hline ACT: & Adoptive T-cell transfer therapy \\
\hline ADA: & Anti-drug antibody \\
\hline ADC: & Antibody drug conjugate \\
\hline BAV: & Biomarker assay validation \\
\hline BLA: & Biologics license application \\
\hline BMV: & Bioanalytical method validation \\
\hline C-Path: & Critical path institute \\
\hline CAR: & Chimeric antigen receptor \\
\hline CLIA: & Clinical laboratory improvement amendments \\
\hline CLSI: & Clinical and laboratory standards institute \\
\hline Cmax: & Maximum concentration \\
\hline COA: & Certificate of analysis \\
\hline COU: & Context of use \\
\hline CP: & Cut point \\
\hline Ctrough: & Trough concentration \\
\hline eCTD: & Electronic common technical document \\
\hline ELISPOT: & Enzyme-linked immunospot \\
\hline
\end{tabular}




\begin{tabular}{|c|c|}
\hline FFP: & Fit for purpose \\
\hline FMO: & Fluorescence minus one \\
\hline FPR: & False positive rate \\
\hline GMP: & Good manufacturing practices \\
\hline GT: & Gene therapy \\
\hline IND: & Investigational new drug application \\
\hline IQR: & Interquartile range \\
\hline ISI: & Integrated summary of immunogenicity \\
\hline ISS: & Incurred sample stability \\
\hline LBA: & Ligand binding assay \\
\hline LCMS: & Liquid chromatography mass spectrometry \\
\hline LLOQ: & Lower limit of quantitation \\
\hline LPC: & Low positive control \\
\hline mAB: & Monoclonal antibody \\
\hline MDB: & Multi-domain biologic \\
\hline MHC: & Major histocompatibility complex \\
\hline MOA: & Mechanism of action \\
\hline MRD: & Minimum required dilution \\
\hline NAb: & Neutralizing antibody \\
\hline NC: & Negative control \\
\hline NTC: & No template control \\
\hline PBMC: & Peripheral blood mononuclear cells \\
\hline PC: & Positive control, used in an immunogenicity assay \\
\hline PCR: & Polymerase chain reaction \\
\hline PD: & Pharmacodynamic \\
\hline PK: & Pharmacokinetic \\
\hline QC: & Quality control \\
\hline qPCR: & Quantitative polymerase chain reaction \\
\hline RBC: & Red blood cells \\
\hline RO: & Receptor occupancy \\
\hline RT: & Reverse transcription \\
\hline SEC: & Size exclusion chromatography \\
\hline SOP: & Standard operating procedure \\
\hline SSCP: & Study specific cut point \\
\hline TBNK: & T cells, B cells and natural killer (NK) cells \\
\hline TE: & Target engagement \\
\hline TI: & Transduction inhibition \\
\hline WRIB: & Workshop on Recent Issues in Bioanalysis \\
\hline
\end{tabular}

The $12^{\text {th }}$ edition of the Workshop on Recent Issues in Bioanalysis (12 ${ }^{\text {th }}$ WRIB) was held in Philadelphia, PA, USA on April 9-13, 2018 with an exceptionally high attendance of over 900 representatives (likely facilitated by the Philadelphia location) from pharmaceutical/biopharmaceutical companies, biotechnology companies, contract research organizations, and regulatory agencies worldwide. The workshop included three sequential main workshop days, two additional advanced specialized sessions and four full-day training sessions that together spanned an entire week in order to allow exhaustive and thorough coverage of all major issues in bioanalysis, biomarkers, and immunogenicity.

As in previous years, this year's WRIB continued to gather a wide diversity of international industry opinion leaders and regulatory authority experts working on both small and large molecules to facilitate sharing and 
discussions focused on improving quality, increasing regulatory compliance and achieving scientific excellence on bioanalytical issues.

The active contributing chairs included Dr Chris Beaver (Syneos Health), Dr Seongeun (Julia) Cho (US FDA), Dr John Kamerud (Pfizer), Dr Steve Keller (AbbVie), Dr Fabio Garofolo (Angelini Pharma), Dr Joseph Marini (Janssen), Dr Adrien Musuku (Pharmascience), Dr Hendrik Neubert (Pfizer), Dr Tim Olah (Bristol-Myers Squibb), Dr Renuka Pillutla (Bristol-Myers Squibb), Dr Sue Richards (Sanofi), Dr Lauren Stevenson (Biogen), Dr Albert Torri (Regeneron), Mr Stephen Vinter (UK MHRA), Dr Jan Welink (EU EMA), Dr Yuanxin Xu (Alnylam) and Dr Eric Yang (GlaxoSmithKline).

The participation of regulatory agency representatives continued to grow at WRIB [1-18], covering topics and discussions in bioanalysis, biomarkers and immunogenicity:

- Regulated bioanalysis: Dr Sean Kassim (US FDA), Dr Sam Haidar (US FDA), Dr Seongeun (Julia) Cho (US FDA), Dr Sarah Rogstad (US FDA), Dr Jan Welink (EU EMA), Mr Stephen Vinter (UK MHRA), Ms Emma Whales (UK MHRA), Mr Gustavo Mendes Lima Santos (Brazil ANVISA), Dr Anna Edmison (Health Canada);

- Biomarkers: Dr Christopher Leptak (US FDA), Dr Shashi Amur (US FDA), Dr Shirley Hopper (UK MHRA), Dr Yoshiro Saito (Japan MHLW-NIHS);

- Immunogenicity: Dr Daniela Verthelyi (US FDA), Dr Yow-Ming Wang (US FDA), Dr João PedrasVasconcelos (US FDA), Dr Brian Janelsins (US FDA), Dr Haoheng Yan (US FDA), Dr Meenu Wadhwa (UK MHRA-NIBSC), Dr Isabelle Cludts (UK MHRA-NIBSC), Dr Pekka Kurki (Finland Fimea), Dr Elana Cherry (Health Canada), Dr Akiko Ishii-Watabe (Japan MHLW-NIHS).

As usual, the $12^{\text {th }}$ WRIB was designed to cover a wide range of topics in bioanalysis, biomarkers and immunogenicity suggested by members of the community, and included daily working dinners and lectures from both industry experts and regulatory representatives, which culminated in open panel discussions amongst the presenters, regulators and attendees in order to reach consensus on items presented in this White Paper.

At this year's WRIB, 39 recent issues ('hot' topics) were addressed and distilled into a series of relevant recommendations. Presented in the current White Paper are the issues' backgrounds, exchanges, consensus and resulting recommendations on these 39 topics, which are separated into the following areas:

\section{LCMS:}

- Small molecule bioanalysis and peptides by LCMS (five topics);

- Small molecule biomarkers by LCMS (four topics);

- Oligonucleotides (five topics).

Hybrid LBA/LCMS:

- Intact biotherapeutic quantification \& PK assays (three topics)

- Immunogenicity assays (four topics);

- Biomarker assays (three topics).

LBA and cell-based assays:

- Gene therapy \& critical reagents (two topics);

- Biomarker assay validation (three topics);

- Flow cytometry (four topics);

- ADA assays \& PK, PD, ADA data correlation (four topics);

- Cut points (two topics).

Key inputs from regulatory agencies:

- In addition to the recommendations on the aforementioned topics, there is a section in the White Paper that focuses specifically on several key inputs from regulatory agencies. 
Due to its length, the 2018 edition of this comprehensive White Paper has been divided into three parts for editorial reasons. This publication (Part 3) covers the recommendations for large molecule bioanalysis, biomarkers and immunogenicity using LBA and cell-based assays. Part 1 (LCMS for small molecules, peptides, oligonucleotides and small molecule biomarkers) and Part 2 (hybrid LBA/LCMS for biotherapeutics and regulatory agencies' inputs) are published in volume 10 of Bioanalysis, issues 22 and 23 (2018), respectively.

\section{Discussion topics}

The topics detailed below were considered as the most relevant 'hot topics' based on feedback collected from the $11^{\text {th }}$ WRIB attendees. They were reviewed and consolidated by globally recognized opinion leaders before being submitted for discussion during the $12^{\text {th }}$ WRIB. Background information on the issues, discussion summaries, consensus and conclusions are in the next section and a summary of the key recommendations is provided in the final section of this manuscript.

\section{Gene therapy}

Viral vector-based gene therapy

What are the major bioanalytical challenges when dealing with gene therapies containing both vector and transgene? What are the bioanalytical method expectations for exposure/ biodistribution? What are the measurements needed to ensure that the gene of interest reached the target cells or tissues? How is efficiency of transfection or transduction in the targeted cells or tissues measured? When should vector shedding be conducted, what is the best bioanalytical assay to use and what are the expectations for assay performance? Is it required for AAV? If using a qPCR assay, is a backup infectivity assay required for AAV? For QPCR assays, are sensitivity requirements the same in preclinical and clinical assays? qPCR assays seem to be key in gene therapy measurements for both biodistribution and vector shedding studies. Is qPCR application possible in regulated bioanalysis and accepted by regulators? Should qPCR become a standard instrument in bioanalytical laboratories involved in performing analysis for gene therapy? What are the key recommendations of validation parameters/criteria for RT PCR used for transgene expression and qPCR used for copy number of vectors vs. BMV guidance/guideline? What are the differences and similarities between qPCR assay validation and traditional LCMS and LBA assays validation? Is immunogenicity on transduced cells really needed? Are vector ADA, anti-transgene and collection of a baseline PBMC sample for ELISPOT enough? For the evaluation of cellular immunity, is ELISPOT the gold standard? What are the ELISPOT assay expectations? Should ELISPOT testing be included as part of routine monitoring during the trial or should samples be banked and tested if symptoms/results from other assays suggest risk of cellular immunity? Is continued monitoring of anti-capsid antibodies and/or NAb required during the course of the trial? What would this data be used for? What should be evaluated for immunity: both anti-capsid and anti-gene product, both ADA and NAb for both capsid and gene product?

\section{Critical reagents}

\section{Critical reagent characterization}

What are the recommendations for establishing a comprehensive and robust program to manage critical reagents? What is the appropriate amount of characterization for critical protein reagents? Is there consensus on the proposal that minimal identity testing needs to be done on all critical reagents, including biophysical analysis, mass spectral analysis and biochemical analysis by analytical SEC? How often and using what type of assays should stability testing be performed? Should forced degradation studies be performed to determine stability? What are the pros and cons when qualifying critical reagents by assay performance and their characterization in later stages? Should orthogonal characterization of critical reagents be required in addition to assay performance for use-date extensions? Are the issues observed with critical proteins in ADA assays primarily related to ruthenium complex (SulfoTAG ${ }^{\text {TM }}$ ) reagents? What are the current approaches for overcoming them? Are formulation conditions currently considered for critical protein reagents? Are there opportunities for a platform for formulation? What are the challenges with externally sourced critical reagents? What can the industry do about poorly characterized or irreproducible commercial reagents? 


\section{Biomarker assay validation}

Biomarker assay validation - ongoing C-Path \& 2017 White Paper in bioanalysis discussions

What are the analytical factors where consensus was not reached at the 2017 WRIB? What is the impact of these key factors on biomarkers assay robustness and reliability? What are the current industry standards/best practices in dealing with the limitations of biomarker assays, such as relative quantitative assays, validation of commercial kits, reagent and kit lot variability, very high/low endogenous levels, specificity, and protein biomarker assay calibrator material? Are there regulatory recommendations for biomarker data utilization? Are these clearly understood and utilized appropriately by the industry? Must the COU drive all aspects of patient biomarker testing? Does this force evolution of biomarker analytics to a clinical endpoint? Do biomarker scientists need a concise FDA or EMA regulatory guidance/guideline for $\mathrm{BAV}$ and/or clinical/pivotal trial data generation rather than rely on best practices?

\section{Level of validation required for biomarkers which are not part of the C-Path White Paper}

What is the industry understanding of the distinction between biomarker qualification vs. biomarker application? Are all biomarkers applied during drug development intended for biomarker qualification? What percentage of biomarkers that drug developers employ during R\&D involves biomarker qualification as the end goal? What is the best industry practice to navigate the complexities of BAV? What considerations are needed for different biomarker types? If the biomarkers of interest are not intended for regulatory decision making, what analytical validation parameters apply (e.g., discovery biomarker translation from preclinical into human clinical studies; biomarkers for exploratory/investigational studies)? What are the primary differences in analytical validation of biomarkers intended for qualification vs. those that are not? What are potential issues/pitfalls to consider when typical bioanalytical laboratory practices are employed (e.g., BMV) with respect to BAV? What is the current status and application of the recommendations in the 2016 \& 2017 White Papers on FFP BAV? Are these recommendations still valid? What are the current practices and ongoing challenges with FFP BAV? Are there any solutions?

\section{Challenges with analytical validation of assays in biomarker development}

It is imperative to have reliable and reproducible biomarker data since the data are often used to make decisions that can impact drug development or an individual's health. What are the key considerations for the development of a robust, accurate, reproducible, reliable, analytically validated biomarker measurement assay? Beyond what has already been recommended in the 2017 White Paper in Bioanalysis, are there any other critical pre-analytical considerations to biomarker development that should be recommended (sample type, sample collection times, sample stability and study design)? What are the current industry standards in considering the biological variability of the biomarker in the populations of interest? What is the best approach for determining the 'normal' level of the biomarker or to determine a cut-off or threshold value? What are the main challenges in using multiple versions of a biomarker assay? How are changes to commercial kits or the use of different assays during biomarker development addressed?

\section{Flow cytometry}

Flow cytometry strengths \& challenges in biomarker assay development \& validation

What do regulators look at when reviewing flow cytometry data? What are the lessons learned from previous challenges in flow cytometry validation? What can be improved: choice of matrix (blood vs. PBMCs), sample collection, assessment of sample stability, better comparison of staining and permeabilization methods? How are the challenges with multiple surface and intracellular markers overcome? What are the approaches to ensure that shared markers across the panels are comparable (staining patterns and intensities)? What is the preferred methodology for cell enumeration: bead-based, reference to TBNK assay, other? What are the challenges with the bioanalytical requirements for CAR-T development?

\section{Current best practices in performing flow cytometry in regulated bioanalysis \& clinical studies}

Are the recommendations outlined in the 2016 White Paper in Bioanalysis still valid? What are the current industry standards in using real-time whole blood analysis vs. batched PBMC? How is the impact of manipulation of PBMC preparation and cryopreservation minimized? What are the current standards in gating strategy: manual gating versus automated algorithm based gating? Centralized versus multi-center manual gating? What are common deviations of the gating strategy? What is the best practice for gating QC? Where possible, should objective gating 
be required in multicolor flow? What is the cut-off of FMO for poorly resolved populations? How should run acceptance criteria be defined? Validation in healthy donor versus patient samples?

Target engagement biomarker assays by flow cytometry

Why is flow cytometry the elective technique for TE assays? Should TE biomarkers (or pharmacodynamic biomarkers) be required for small molecules in early stage studies? How should the validation of TE biomarkers be approached? Assay performance in a lab often depends upon the precision of the operators and the protocol. Should emphasis be placed upon the process rather than assay validation performed before the trial? Is a FFP validation in this context appropriate given that although assays can be variable, the central tendency of the data provides meaningful information for a drug development team? Flow cytometry assays for TE are typically relative assessments - that is, each time point is relative to the baseline or pre-dose time point. When performed under controlled conditions, these can be very reproducible but assay development and validation procedures often do not account for this. What is appropriate validation for the effect of time and sample dependency?

\section{Challenges with receptor occupancy assays by flow cytometry}

What should be evaluated during assay development of flow-based RO to assess RO in target tissue? What should be included in a FFP validation of flow-based RO? What is the industry standard to approach LLOQ validation $\mathrm{RO}$ measurement by flow cytometry? When is LLOQ validation needed? Is it needed for RO assays? Should the variation in $\mathrm{RO}$ where the expected result is $100 \%$ be quantified - that is, where excess drug is present? Typically, the goal is to maximize RO for some duration. What is the LLOQ for unbound receptor? What is the necessity of absolute versus relative quantification of RO? Is there the need to provide further clarification/harmonization of the terms: qualification vs. validation; FFP validation vs. iterative approach; BAV vs. analytical validation? The CLSI committee appears to support the use of the term quasi-quantitative. In the context of the seminal Lee et al. paper [19], the term makes sense and the concept justifies how to approach flow validation. What are the pros/cons of the usage of this term?

\section{ADA assays \& PK, PD, ADA data correlation}

$P K, P D, A D A$ data correlation \& the merit of $N A b$ assays

Is it possible to make the same conclusions from $\mathrm{PK}, \mathrm{PD}, \mathrm{ADA}$ data correlation without standalone $\mathrm{NAb}$ assay data? What assay(s) are industry professionals leveraging to understand presence and impact of NAbs in addition to, or in lieu of, standalone NAb assays? Have regulators seen examples where assays other than standalone NAb assays were deemed suitable for understanding NAb impact? Since assay performance is measured using PC in validation and PC are frequently not representative of genuine responses in clinical studies, when regulators are reviewing the suitability of an assay, how much consideration is given to the assay's ability to identify clinically meaningful immunogenicity in-study as opposed to the performance of the PC in validation? Is there a need for standards (e.g., PC) and standardized assays? Is there a demand for standards? If standards were available, would they be used?

\section{Understanding clinical relevance of $A D A / N A b$}

How should we define clinical relevance of ADA for risk assessment: by incidence of ADA/NAb, by percent of patients exhibiting negative effect of ADA/NAb, by the observed extent of decrease/increase in clinical endpoint, or other? Are the data from clinical development programs generally adequate to inform/assess the clinical relevance of ADA? In your experience, how often is PK useful for detecting/measuring ADA impact and for what type of products? Are there any special considerations (enabling factors)? We talk about clinically relevant ADA, but that indicates there is also clinically non-relevant ADA. Can we use such categories broadly when describing a product or should it be limited within the context of individual trials? Does this also indicate that we should always do further analysis of ADA response data, not just ADA status (positive vs. negative)? Most agree that persistent treatment emergent $\mathrm{ADA}$ responses can lead to clinical consequences. There are likely also thresholds where one sees a clinical impact. How could one approach determining a clinically relevant ADA titer/threshold? Are there aspects from the assay and bioanalysis perspective that we should consider to facilitate this? What is the view in reporting final titers for clinical settings? Should all dilutions be factored in the calculation resulting in a titer for neat sample or can one report assay titer with a note as to MRD - among others? How do you decide which NAb methodology is best for determining clinical relevance: enzyme activity-based, target binding (LBA format; cell-based), or cellular uptake 
(LBA; cell-based)? When should multiple NAb assays be required for development? What analytical approaches work best for determining clinical impact of ADA: subgroup analysis by patients with altered PK, PD, safety events or reduced efficacy; subgroup analysis by ADA status: positive vs. negative, titer magnitude, quartile, top $10 \%$ vs. bottom $10 \%$, persistent vs. transient? Is it reality or a myth that all NAb have a negative clinical impact? If no clinical impact is found, is there an issue with the selected NAb assay, length of time patients were followed in the clinical trial, and/or flawed analyses were performed? How is it possible for there to be no clinical impact of a high-titer NAb?

Isotyping \& domain/ epitope mapping of ADA: utility of ADA isotyping assays?

How has ADA IgE isotyping data helped predict and manage drug induced anaphylaxis reactions? Why is isotyping $\&$ domain/epitope mapping requested by regulators and how often? Could we consider the removal of the recommendation to screen for IgA and IgE unless there is a specific reason?

Strategies, regulatory expectations \& clinically meaningful $A D A$ assays

What are the risks associated with changing PC during assay evolution and what considerations should be taken into account: CP drifting in post-marketing ADA monitoring? Maintaining continuity and comparability of immunogenicity data? Bridging ADA assay changes over a clinical development history? What are the strategies and clinically meaningful ADA assays for monitoring ADA in patients treated with approved products? What criteria should drive the monitoring: decisions impacting patient safety; decisions impacting drug effectiveness under 'real world' clinical settings? Where are the samples for ADA monitoring analyzed, in a regulated bioanalytical laboratory or a CLIA accredited lab? What types of methods are used to analyze these samples: a laboratory developed test or a diagnostic assay?

\section{Cut points}

Issues in cut point determination \& $A D A$ assay drug tolerance \& sensitivity

Analytical variability determines minimum screening and confirmatory CP values. Current procedures recommended for CP determination (e.g., outlier exclusion) may result in CP that are too low based on acceptable analytical variability. Using CP below the minimum dictated by the analytical variability is likely to give immunogenicity results with diminished clinical relevance. Is a minimal CP rule based on the assay variability of the negative control (NC) defendable and acceptable? Are the "supersensitive ADA assays" $(<10 \mathrm{ng} / \mathrm{ml})$ a problem for the industry? Does the PC performance need to be taken into consideration? Is assay variability really a determining factor? If so, does it indicate that the assay is not robust enough and should be further optimized? What is more critical: assay variability or sample variability? What is the significance of a validation parameter like stability? Would ISS not be a better option? What PC concentration should be used to establish drug tolerance and sensitivity limits? ADA assay sensitivity is related to the affinity of the surrogate PC antibodies used in the assay. Is there an upper limit of affinity for surrogate ADA PC? What are the regulators' expectations regarding drug tolerance for ADA assays - for example, steady state $\mathrm{C}_{\text {trough }}$ or $\mathrm{C}_{\max }$ of the expected drug concentration for the anticipated high dose level? Why is it not always possible to develop an assay with adequate sensitivity/drug tolerance? Is this due to limitations in assay technology, product, matrix and/or posology? Does the requirement for sensitive and drug-tolerant assays modify the current concept/paradigm of assay development? With 'supersensitive ADA assays', is it relevant to detect ADAs at this level? Is it necessary to have 5\% FPR (why not 1\% FPR - need flexibility in guidance/ guideline) especially when the calculated CP is too low based on the analytical variability? In the 2017 White Paper in Bioanalysis, regulators indicated they are looking for a $1 \%$ failure rate for the low positive control (LPC). Is the LPC concentration selected by targeting a two-to three-fold signal to noise ratio or by determining it statistically using the validation data? How is the expectation handled for the $1 \%$ failure when dealing with different $\mathrm{CP}$ values for different disease populations or different studies? Is it really practical to expect a $1 \%$ failure rate when $\mathrm{CP}$ have to be adjusted or re-established? Do methodologies to increase drug tolerance negatively impact ADA detection? Following the 2017 White Paper in Bioanalysis [18], has drug tolerance become more important than sensitivity? Based on the above, when choosing a NAb assay format what would the priority be between a cell-based assay with poor drug tolerance and a LBA with better drug tolerance? Do more sensitive and drug tolerant $\mathrm{ADA}$ assays detect more clinically irrelevant signals which confound evaluation of ADA? Are validation cut points being established using naïve samples or commercially obtained samples from disease populations right from the start? If using commercial disease samples, how often are these samples really similar to the actual study 
population? How often are normal distributions with samples from disease populations during CP determination detected? Is checking for normality really needed? Is an appropriate CP for a population or a study determined by initial analysis of baseline samples (prior to actual production runs) followed by confirmation that about 5\% false positives from these samples are generated? Is this a statistical approach or just rule of thumb? Based on the collective industry experience, how often are study-specific CP needed? Is high variability observed across different studies, even within the same disease population?

\section{Issues with dynamic, NAb, biosimilar cut points \& outliers}

Are there any cases when a dynamic CP would be the preferred path forward, or do they indicate that a method is sub-optimal? Is setting a confidence interval around acceptable false positive rates an appropriate guide when an in-study CP would be appropriate? What parameters must be in place to generate an in-study CP with sufficient confidence to implement it in place of the pre-study, validated $\mathrm{CP}$ ? What is the best way to identify and remove biological outliers/pre-existing positives from CP determinations? Box plot based on sample medians (biological outliers)? Are there other statistical approaches being used to identify and exclude outliers during CP determination? Beyond the symmetric boxplot with $3^{*} \mathrm{IQR}$, how common is the use of an adjusted boxplot for skewed data sets where the distribution is influenced by technical limitations (very sensitive methods)? Is a $\geq 50 \%$ Tier 2 inhibition outlier rule reasonable and acceptable for removing pre-existing positives? Based on the 2017 White Paper in Bioanalysis recommendations, is it now possible to reach a consensus regarding use of outlier factors during the determination of CP? Has the industry moved to an outlier factor of 3 (i.e., $3^{*} \mathrm{IQR}$ )? If so, how has this change impacted the immunogenicity rates reported? Did we see a better correlation between immunogenicity rates and PK impact/efficacy? What is the current industry standard for setting up CP and data reporting in presence of pre-existing reactivity? Evolution of response over time within a subject, rather than on absolute 'positive/negative' values per se? How is the pre-existing seroreactivity addressed during development of immunogenicity assays for vaccines? Is there a need for clearer vaccine specific regulatory guidelines?

\section{Discussions, consensus \& conclusions}

\section{Gene therapy}

Viral vector-based gene therapy

In recent years, a growing number of pharma and biotech companies have been involved in development of viral vector-based gene therapy (GT) modalities. Regulatory agencies have also recognized the importance of this field by releasing several documents over the last two decades [20-27].

Viral vector GT treatment modalities pose unique safety and bioanalytical challenges that can vary based on the type of vector used, the properties of the transgene, as well as the route of administration and target cell population. Factors which must be considered in the development of the bioanalytical strategy include preexisting immunogenicity [28], the development of humoral and cellular immune responses to both the vector and transgene [29], strategies for the evaluation of PK and PD responses, and the risk of viral shedding and environmental exposure [30].

On target and off target tissue transduction are critical elements of safety risk assessments [31]. Monitoring the drug moiety - that is, vector and transgene, or transgene alone (depending on which type of vector is used) - is done to determine exposure in clinical studies. It is recognized that biodistribution studies are difficult to perform in a clinical setting, due to tissue biopsy limitations. In such cases, non-clinical studies can provide biodistribution information. However, the translation of this information from animal to human may depend on the vector or mode of delivery of the transgene as well as the transgene itself and the host disease state [32].

Exposure and biodistribution are typically determined by qPCR, although other technologies are available (e.g., imaging, bDNA, RNA-seq), with normalization to an appropriate host reference gene. For the most part, tissue assays would be necessary; however, obtaining appropriate samples is a major challenge. Expression of the transgene (PD) may be measured by RT-PCR, or by detection of expressed protein by LBA or LCMS. For each GT modality, an assessment of bioanalytical challenges and strategies should be outlined and assessed at each phase of drug development.

With viral-based vectors, characterization of vector shedding is critical to minimize the risk of disseminating infectious material [22,33]. Matrices that are typically evaluated for viral shedding include urine, saliva, feces, and semen; however, the matrices selected should take into account the tropism of the vector and the route of administration. Total shed vector can be determined by qPCR. If present, the potential infectivity of the shed virus 
is determined by a cell-based infectivity assay (bioassay). Viral infection assays may be difficult to perform and can be challenging with certain matrices such as urine [34]. In those instances, a surrogate marker may be used to monitor the infectious titer present by staining for a late stage viral replication protein in a cell based assay, a read-out which typically requires $24-48$ hours. Cell lysates and fixed cells may also be considered, in-lieu of an infectious particle assay.

The application of qPCR assays is key in gene therapy measurements for both biodistribution and vector shedding studies and data generated by PCR would be submitted to the regulators. Hence, bioanalytical laboratories involved in performing analysis for gene therapy should consider qPCR as a standard assay platform in their toolbox.

Although there are no specific guidance/guidelines for PCR validations, US FDA requires the use of a wellcharacterized assay. Depending on the GT modality or transgene elements (i.e., tissue specific elements or viral tropism or serotype), the sensitivity requirements may be different in preclinical vs. clinical assays. Assay performance controls and QCs should be established to monitor assay performance. Validation parameters are similar to those for LCMS and LBA validations such as accuracy, precision, range of quantification, analytical sensitivity - among others. NTC should be negative and primers should be specific to the viral vector and transgene. Sensitivity can be established based on the number of copies detected per microgram of genomic DNA. Frequent and early interaction with regulators is recommended.

Pre-treatment immunogenicity to the viral capsid may impact safety and efficacy of the therapy. Specifically for a systemic administration, anti-capsid NAb to viral vector (transduction inhibition or TI) testing should be evaluated as a potential enrollment criterion. Depending on the prevalence of pre-dose anti-capsid NAb, criteria for enrollment exclusion (or inclusion) needs to be carefully assessed especially in the case of high prevalence of pre-dose NAb. There was no consensus on the value of total anti-capsid Ab assays. For engineered T cells expressing a CAR, detection of anti-CAR antibodies may be useful in interpretation of PK or efficacy data.

Cellular immunity should be characterized by detecting potential cytotoxic T-cell responses. For the evaluation of cellular immunity, ELISPOT for T-cell cytokines (such as IFN $\gamma$, IL-2, etc.) is commonly used, although other methods such as intracellular cytokine analysis by flow cytometry are being investigated. It should be recognized that ELISPOT and flow cytometric assays are challenging to perform and no guidance on their validation is available. System controls should be implemented to ensure assay performance is consistent. Harmonised processing and storage of PBMC samples at each clinical site is critical to ensure consistent cell viability and functionality. If not, these functional assays will be compromised. Cellular, NAb and ADA responses to transgene were considered to be important, but monitoring the efficacy of the therapy is the most relevant, clinically. ADA and NAb to gene product was also discussed. The consensus was to monitor the anti-product ADA and if there is concern over spreading of immunogenicity to endogenous proteins, an assessment of neutralizing antibody activity may be warranted.

\section{Critical reagents \\ Critical reagent characterization}

The need to understand the quality of critical reagents that go into a LBA is not a new concept. The robustness of bioanalytical data is reliant upon a clear understanding of the assays used to generate the data. The ability to fully characterize all biologic reagents through epitope mapping, biophysical and biochemical measurements enables deeper knowledge of assay readouts and provides greater confidence in the data generated from those assays. Scientists understand that using biological reagents to indirectly measure an analyte of interest has challenges, particularly in verifying the species that are being detected. The verification of the binding interaction (biophysical characterization) is often as critical as the validation of the assay itself.

Critical reagents are generally produced using a biological (and in some cases, an additional chemical) process that makes them inherently prone to lot-to-lot variability, which can impact performance of bioanalytical assays. Vendors may change lots or the identity of their product without notification and some information on the reagents may be withheld as proprietary. Therefore, maintaining lot-to-lot consistency can be challenging and careful characterization to understand the unique characteristics of these reagents is crucial for consistent assay performance.

Critical reagents for each method should be clearly identified. A comprehensive and robust program to handle critical reagents should include SOPs or work instructions for preparation, qualification, labeling and storage. Defining the critical attributes of required reagents and having a testing strategy for characterization of key attribute determinants is recommended. Often, this involves having a certificate of testing or analysis for physical 
characterization in addition to functional testing within the assay. Implementation of an electronic inventory system is recommended to track inventory and expiry/retesting of all reagents.

The appropriate amount of characterization for critical reagents was discussed and the recommendations from the 2015 White Paper were upheld [12]. The identity of the reagents should be established. Concentration, binding activity, purity, labeling efficiency, and freeze-thaw stability are other key determinants. Although characterization of a reagent based on assay performance is necessary, orthogonal characterization based on physical attributes might be recommended depending on the reagent type and intended use [35].

Stability can be assessed based on performance within the assay which should be maintained throughout the life-cycle of the product. Freeze-thaw stability is highly recommended, as reagents are typically shipped in a frozen state. It was recommended to retain several aliquots of key reagents to ensure bridging experiments and possibly for troubleshooting efforts when necessary [36]. Forced degradation studies are not recommended for critical reagents.

The most common format of ADA assays requires biotinylated and ruthenium complex reagents. Ruthenium complex reagents can be prone to aggregation [37], which can impact ADA assay performance, and therefore SECbased characterization is recommended. Formulation conditions can impact stability and aggregation, especially during freeze-thaw [38]. For example, $\mathrm{pH}$ can influence aggregation. The addition of sucrose or trehalose may help to minimize the impact of freeze-thaw on aggregation, but no universal formulation has been identified.

In general, characterization should be performed on critical reagents. However, biophysical testing to the degree of GMP standards may not be practical for all critical reagents. When possible, it is an important way of better understanding the reagents and thus the assay.

\section{Biomarker assay validation}

Biomarkers can have multiple applications in healthcare, including use as drug development and diagnostic tools. It is imperative to have reliable and reproducible biomarker data in these settings, since the data are often used to make decisions that can impact drug development or an individual's health. The need for application of biomarkers to accelerate drug development is a widely acknowledged topic within the pharmaceutical industry and the regulatory community. Together with this recognition, there has been an increased focus on application of biomarkers during preclinical and clinical development.

Due to the complexity of considerations associated with biomarkers, the path to defining specific requirements for assay development and validation of biomarkers is not a one-size fits all solution. This path is complex and the course depends on the biomarker category and the context of use of the biomarker.

\section{Biomarker assay validation - ongoing C-Path \& 2017 WRIB White Paper discussions}

Over the last several years, the FDA as well as others in the scientific community have co-sponsored multiple workshops aimed at advancing the discussion around BAV [12,15,18,39]. Of particular importance are the analytical factors that must be considered when assessing the robustness and reproducibility of a biomarker assay used to qualify a biomarker. Under the leadership of the Critical Path Institute (C-Path), the Biomarker Assay Collaborative Evidentiary Considerations Writing Group has developed a draft framework outlining key criteria and best practices for biomarker assay performance expectations and validation. On June 2017, a two-day public workshop was convened at The Duke-Margolis Center for Health Policy (DC, USA) that served as a forum for broader input and feedback on this framework, to guide the establishment of a comprehensive set of harmonized best practices and performance characteristics for BAV for the purpose of biomarker qualification intended to be used as a drug development tool $[40,41]$.

Biomarker qualification (with the exception of most target engagement PD biomarkers) is independent of the specific drug or product. Once established for a specific COU, the biomarker can be used without re-review for other drug products across multiple drug development programs. The C-Path White Paper recommendations on analytical validation are therefore not intended for a specific drug development process, rather they are intended for the qualification of biomarkers independent of the drug under investigation.

As a follow up to C-Path \& 2017 White Paper in Bioanalysis recommendations, it was proposed that a concise regulatory guidance/guideline for $\mathrm{BAV}$ is desirable rather than the industry relying on best practices [18,41]. Moreover, it was strongly reconfirmed by both industry members and regulators that the COU must drive all aspects of patient biomarker testing, resulting in an iterative process where multiple cycles of assay validations are likely. Because there are no detailed and clear regulatory recommendations for BAV, it is recommended that developers consult the regulators early in the development program to ensure the proper testing strategy is implemented, especially 
if the biomarker is a clinical endpoint or intended to inform on an endpoint. Current consensus in the C-Path document points scientists to follow the FNIH Biomarkers Consortium Framework for Evidentiary Standards for Biomarker Qualification [42].

\section{Level of validation required for biomarkers that are not part of the C-Path White Paper}

Consensus between industry and regulators was reached regarding the importance of clarifying the major difference between the predictive biomarkers that are eventually targeted for decision making at the patient level and other categories of biomarkers, as defined in BEST [43], applied during R\&D phases of drug development. Biomarkers can be used for multiple purposes, such as exploratory; dose-response evaluation; PK/PD modeling; and for internal decision making based on effective target engagement, achieving proof of biology/mechanism, proof of concept, and indications of efficacy and safety. The assays intended for biomarker qualification, which currently constitute a very small fraction of biomarkers that will be submitted for use in drug development, must be analytically validated and fall under the scope of the C-Path White Paper [41]. Ideally, there would be a road map available that determines the level of validation necessary for biomarkers which are not intended for qualification. It is recommended that this roadmap indicate that the assay must be adequately validated for the COU if a biomarker is used for regulatory or patient management decisions, such as patient selection or treatment allocation.

Key concepts related to biomarker assay development at various phases of drug development include an understanding of the reference standard characterization required (when NIST, WHO or USP standards are not available); understanding the difference between endogenous biomarkers and the calibrator material used in assays; key parameters such as parallelism, relative accuracy, precision, stability, sample integrity; and finally, the use and interpretation of biomarker data (qualitative, relative quantitative versus quantitative) - among others [44].

If the biomarker of interest is not intended for regulatory decision making, one must first establish COU and let that determine the FFP BAV [19]. FFP BAV recommendations were previously issued in the 2015-2017 White Paper in Bioanalysis $[12,15,18]$, and it was confirmed that they are still valid. It is crucial that BMV guidance not be applied to BAV in a prescriptive or overly rigid fashion to ensure that the appropriate scientific rigor is applied for each specific COU.

Subsequent to the WRIB meeting, the FDA released the revised BMV Guidance [45]. This guidance addresses $\mathrm{BAV}$ and states "Biomarkers can be used for a wide variety of purposes during drug development; therefore, a FFP approach should be used when determining the appropriate extent of method validation. When biomarker data will be used to support a regulatory decision making, such as the pivotal determination of safety and/or effectiveness or to support dosing instructions in product labeling, the assay should be fully validated." This guidance is consistent with the recommendations above.

\section{Challenges with analytical validation of assays in biomarker development}

Development of a robust, accurate, reproducible, analytically validated biomarker assay/test is critical for development of biomarkers. Analytical validation is the process of "establishing that the performance characteristics of a test, tool, or instrument are acceptable in terms of its sensitivity, specificity, accuracy, precision, and other relevant performance characteristics using a specified technical protocol (which may include specimen collection, handling and storage procedures)" [43]. Analytical validation for the purpose of biomarker qualification includes establishing that the analytical performance characteristics of a biomarker test, such as the accuracy and reproducibility, are acceptable for the proposed COU in drug development.

Pre-analytical considerations including sample type, sample collection times, sample stability in biological matrices, sample shipping conditions and study design, are critical to biomarker assay development. Changes in sample type or collection times can be mitigated by re-performing validation with the new samples. Bridging studies may also be an option. Sample stability in biological matrices should be determined over time. The duration of time assessed should encompass the anticipated duration of sample storage from time of collection to time of analysis. It is recommended to address sample stability beginning from assay development in order to ensure that established sample stability covers the needs of future studies.

Biological variability of the biomarker in the populations of interest is also an important consideration. In the drug development realm, rarely there are established standards for determining the 'normal' level of the biomarker or a cut-off or threshold value. Data generated is typically expressed in relative quantitative terms and evaluated by comparing baseline values to post-treatment values over time or by comparing values from placebo versus dosed groups. In general, the approach would be to first generate a hypothesis, then validate the assay, gather the data 
and interpret the data in collaboration with the statisticians. Cut-off or threshold values should be determined using a training dataset and confirmed using a separate validation dataset. As more data are collected and with each iteration of assay validation, the cut-off value may need to be refined.

Using multiple versions of an assay can also present challenges. For example, the extent of bridging studies required may be different depending on if the assay change occurs within or between studies. Changes in commercial kits employed as part of the assay should be minimized, but if unavoidable, then bridging studies are required [46,47]. These may be difficult to execute if the assays are very different (e.g., direct vs. functional assays).

Most importantly, it is essential to understand the purpose and clinical requirement of the assay as they relate to the biomarker's COU before deciding on the criteria for performance and rigor of criteria for the assay validation.

\section{Flow cytometry}

Flow cytometry strengths \& challenges in biomarker assay development \& validation

Flow cytometry is a powerful tool for multi-parameter analysis of a single cell within a heterogeneous cell population using fluorescence-based detection of the cell surface and/or intracellular antigens. It allows for detailed characterization of phenotypes and functions of cell subsets in ways that are not possible using bulk assays and is utilized widely for monitoring immune status in clinical studies. While clinical trials may involve a single research site or multiple global research sites, system immunology studies often require the analysis of flow cytometry data from multiple clinical trials acquired by different research sites. Efforts at standardization have been proposed, including identifying the appropriate quality controls and developing standardized phenotyping markers, which would allow for integration of data from various clinical studies [48]. Even with standardized phenotyping markers, antibody clones and fluorochromes with appropriate titrations still need to be tested and optimized empirically for each assay developed and validated.

Flow cytometry-based biomarker assessments in drug development can provide critical information to demonstrate target engagement, pharmacodynamic responses, and potentially predictive signatures of therapeutic efficacy and safety responses. There are a wide variety of flow cytometry assays implemented in clinical studies such as cell enumeration, multi-parameter immunophenotyping, receptor occupancy and cell signaling. For cell enumeration, calculating cell numbers by referencing to counts from complete blood count analysis will add more variability than using a single platform bead-based methods [83,84].

As flow cytometry assays lack the availability of reference standards and the data generally fall into the category of quasi-quantitative, it is thus not possible to validate accuracy in the traditional manner. Rather, alternative approaches to establish reproducibility have been described by the International Clinical Cytometry Society that provide confidence in the assay results [49]. When antigen expression levels are quantified using fluorescence quantitation beads, resulting data would be considered relative quantitative given that the beads serve as a fluorescence intensity calibrator but they are not fully representative of the test material $[49,50]$. Flow cytometry assays can also generate results which are qualitative, when the presence or absence of an antigen is reported or when the assay is used for the characterization of a hematological malignancy. Selection of the assay format and data output should be based on the COU of the biomarker.

Conducting longitudinal flow cytometry assays as part of multicenter clinical trials poses several challenges. Proper assay development and optimization should be used to mitigate these challenges; an optimal workflow should be established and controls should be implemented in order to ensure data quality over time and inform data interpretation. Critical reagents (e.g., permeabilization buffers, antibody reagents) and procedures should be well characterized during development and validation, and properly controlled during sample analysis.

Flow cytometric testing often occurs as real-time sample analysis of fresh blood without preservatives. To monitor assay performance during longer durations of real-time testing, one strategy to consider is incorporating analysis of QCs from the same cell bank (either cryopreserved or fixed cells). The applicability and decision-making related to such assay QCs should be established during method development and validation. For multi-lab sample analysis, lab-to-lab comparability should be established to ensure consistency for sample processing, testing, gating/data analysis, and training. Use of comparability samples (similar to proficiency assessments by CLIA labs) could be considered. The use of banked PBMCs for sample analysis is less preferred, as many challenges exist with preserving cell viability and accurate expression of certain cell surface antigens.

As with any biomarker assay, a FFP approach will guide the specific validation parameters assessed for cell-based biomarkers representing exploratory, PD and predictive endpoints in a clinical study. Scientifically-driven FFP 
validation parameters for flow cytometry assays should typically focus on establishing the specificity of critical reagents; intra- and inter-batch assay precision; sample pre-analytics and stability; understanding biological and longitudinal variability of the biomarker of interest; and thresholds of sensitivity. When appropriate, potential interferences related to the specific patient population should be characterized, reference ranges established for clinical decision-making, and biologically relevant quality controls developed to monitor assay performance.

Consistent sample quality is a fundamental challenge in flow cytometry analyses, as the sample is comprised of living cells that are sensitive to a multitude of pre-analytical factors. The biomarker scientist and analytical lab should develop SOPs outlining sample collection, handling, and shipping procedures, including temperature controls. These SOPs will need to be implemented across clinical sites to ensure consistent sample quality and stability across the study. It is important to understand that the collection of samples at the clinical site(s) is a critical step to ensure high quality, valid data, thus implementing training, detailed instructions in laboratory manuals, and appropriate auditing of the site's practices can help to ensure sample quality.

\section{Current best practices in performing flow cytometry in regulated bioanalysis \& clinical studies}

A series of recommendations regarding the validation of biomarker assays using flow cytometry have been issued $[15,49,51]$. Based on the findings during assay validation, a FFP approach for run acceptance criteria based on critical assay parameters or sample acceptance criteria should be used. For example, these criteria may include sample stability thresholds, the number of minimum events collected, quality of RBC lysis, and established QC criteria (if using).

Flow cytometry assays can be deployed using real-time whole blood analysis as well as batched cryopreserved PBMC. Real time sample analysis is scientifically ideal, but does require method development and validation prior to initiation of study sample analysis. In some cases, it may be advantageous to establish a window for sample analysis - for example, batch testing of all samples between 48-60 hours (actual timing to be determined in validation) rather than processing samples when received. While using cryopreserved PBMC offers the opportunity to batch test samples at a single testing lab, there can be some biological limitations due to the impact of PBMC preparations and cryopreservation - for example, poor recovery of some subsets, modulation of cell-surface markers such as chemokine receptors and activation markers, and reduced cell yield and cell function after freeze-thaw of the sample. To minimize the impact of these issues, it is recommended to implement rigorous testing and validation of marker stability, training on PBMC isolation and cryopreservation and monitoring of the sample handling and post-thaw sample quality.

During sample analysis, implementing a gating strategy and template is a critical part of the assay that should be initially defined during method development and validation, but may need to be refined and optimized in-study. Critically, there was consensus that gating strategies should be driven by a rules-based approach, which limits the impact of subjectivity across analysts and ensures consistency - that is, use rules-based gating when determining the cut-off of FMO for poorly resolved population. Quality control review of the gating is required and any changes should be properly documented.

Assay performance often depends upon instrument calibration, operator expertise and consistency of executing the SOP. This is not unique to flow cytometry as any assay would need to assess operator and instrument variability. Cross-validation should include precision, qualification of operators and instruments at secondary locations, but repeating sample stability assessments is not required.

It was discussed if healthy donor versus disease samples should be analyzed during method development and validation. The recommendation was to use a scientific rationale based on a FFP approach. In some cases, disease samples may not be needed to guide assay design and validation but can be evaluated as disease samples become available in-study. In other cases, disease samples may be needed to establish criteria for the clinical study - for example, for hematological malignancy or for activation markers uniquely expressed in disease. There was consensus that when disease samples are available, they should be evaluated and will ultimately help inform biomarker utility and data interpretation.

One of the most promising recent developments in cancer immunotherapy is CAR T-cell therapy, a form of ACT, in which autologous or allogeneic $T$ cells are removed from the patient or donor and genetically engineered to express receptors against tumor-specific surface antigens. The modified cells are then cultured in vitro to greatly expand their numbers and then reinfused into the cancer patient, where they bind to tumor cell surface antigens in non-MHC restricted fashion, proliferate and kill the tumor cells. So far, two CAR T-cell therapies have been approved in the US in 2017 and a growing number of CAR T-cell therapies are under development by multiple 
pharmaceutical companies. Monitoring the infused CAR T cells can be considered 'cell-based PK', and is crucial to evaluate the post-antigen exposure-related expansion and subsequent persistence. While qPCR is the primary bioanalytical method for PK measurement of CAR-T therapy, flow cytometry is used to monitor the expression of CAR antigen on circulating CAR T cells and also the cellular kinetics of CAR T cells [52-54].

There are several challenges to developing an appropriate bioanalytical method, in terms of sample type, reagents, and cellular analyte stability. One solution is to generate an appropriate CAR-expressing T-cell for method development from healthy donors. Furthermore, custom reagents for CAR detection are needed with appropriate life cycle management plans.

\section{Target engagement biomarker assays by flow cytometry}

The term TE refers to either RO assays for large molecules or describes ex vivo cellular or biochemical assays used to quantify the degree of inhibition upon cell stimulation. Flow cytometry assays for TE are typically relative assessments - that is, each time point is measured relative to the pre-dose time point. When performed under controlled conditions, these can be highly reproducible. FFP assay development and validation procedures should account for this and include parameters around biological variability, longitudinal variability, and potential interferences. Whole blood flow cytometry is recommended because it limits manipulation of the sample and maintains therapeutic drug levels in the sample, allowing for the most accurate assessment of target engagement.

A key challenge in developing novel small molecule immuno-modulators is that the targeted pathways are active only in small numbers of specific cells which are generally inaccessible. When larger numbers of cells that are capable of responding to the desired pathway are present in peripheral blood, they can be stimulated in the lab after the donor has been dosed with the compound - that is, ex vivo stimulation of the pathway of interest. Ex vivo stimulation of whole blood can be a valuable tool used to assess the activity of a drug in the clinic and flow cytometry is an ideal analytical platform for these analyses because specific cell types can be queried in a variety of matrices with relatively high throughput.

Various approaches can be used to induce a marker specific for the pathway of interest in a relevant cell type. Typical markers include phosphorylated proteins in the targeted pathway, intracellular markers translocated to the cell surface or transcriptionally regulated marker expression - that is, phosphorylated STAT proteins, surface CD63, and expression of CD69, respectively.

Target engagement data can provide a good approximation of the potential target inhibition which can be used to guide dose escalation decisions, to ensure complete coverage of the exposure response curve and to better understand when inhibition has been saturated. These data can guide first-in-human trials and although not a requirement, TE biomarkers (or PD biomarkers) can be valuable to inform the PK/PD relationship. In patient efficacy trials, TE biomarkers can then confirm that the desired biologic effect was achieved. These data are especially important when the compound does not have the desired clinical efficacy. As important tools in drug development used to facilitate different decisions, validation of TE biomarkers should be FFP based on the context of use and characteristics of the assay as described above.

\section{Challenges with receptor occupancy assays by flow cytometry}

Receptor occupancy assays are designed to quantify the binding of therapeutics to their targets on the cell surface and are frequently used to generate PD biomarker data during nonclinical and clinical studies of biopharmaceuticals. When combined with the PK profile, RO data can establish PK/PD relationships, which are crucial for informing dose decisions. $\mathrm{RO}$ is commonly measured by flow cytometry on fresh blood specimens and is subject to numerous technical and logistical challenges. To ensure that reliable and high-quality results are generated from RO assays, careful assay design, key reagent characterization, method validation, implementation in studies and data reporting/interpretation, are of critical importance. The investigator needs to understand what cell type to measure (based on MOA), as well as the RO profile (free, bound, or total, the measurement of each may be limited by reagent availability). The approach that best fits the method and context of use should be used (direct and indirect are both viable options). Flow cytometry is not generally recommended for tissue RO due to technical challenges that are likely to impact the integrity of the target and the dissociation of the drug [17]. If this avenue is pursued, the described challenges should be thoroughly assessed during method development and evaluated for feasibility of implementation.

Literature is available that outlines what should be included in a FFP validation of flow-based RO assays [55-57]. The discussion at this year's WRIB was to determine the industry standard to approach LLOQ validation. It was 
concluded that the target needs to be detectable with appropriate precision; more critically, a RO window with sufficient dynamic range should be established during development. The imprecision of the measurements should be less than the biological variability. Determination of maximum inhibition (100\% receptor occupancy) by spiking in excess drug is recommended.

Further discussion is needed in order to agree on the terminology to use in flow-based assays - for example, qualification versus validation; FFP validation versus iterative approach; BAV versus analytical validation. Several discussions have already begun [16,58], but no consensus has yet been reached between industry and regulators. The Clinical Laboratory Standards Institute is in the process of issuing a guideline on the validation of assays performed by flow cytometry (CLSI H62), which can be leveraged in designing FFP assay validation. This document will support the use of the term quasi-quantitative as described by Lee et al. [19], providing justification as to why traditional approaches to accuracy validation cannot be applied to flow cytometric method validation.

\section{ADA assays \& PK, PD, ADA data correlation}

All ADA have the potential to influence the exposure, efficacy, and safety of protein therapeutics [59,60]. Knowledge and practices regarding assessment of immunogenicity of biotherapeutics have significantly advanced over the past decade. Factors that influence the development of ADA are better understood, approaches to measure ADA are increasingly harmonized and a risk-based approach is used to develop a clinical immunogenicity assessment program. An ongoing challenge facing immunogenicity professionals is the interpretation of the clinical impact of ADA. Discussions within the immunogenicity community have focused on whether overall ADA incidence or more detailed reports on ADA titers and the link with clinical consequences may be more informative. The overarching goal is to ensure that meaningful, clinically relevant information is provided to physicians involved in treating patients.

Immunogenicity risk assessment for IND support is currently governed by the 2014 FDA Guidance [61] and 2016 FDA draft guidance [62], which requires the analysis of product/CMC, patient and treatment related factors. It was recommended that a risk-based approach, which is also advocated in the immunogenicity guidance from EMA [63], is needed to balance the potential harm with potential benefits of a new biotherapeutic throughout clinical development based on the likelihood of developing an immune response, the risk of a response to the patient, the reversibility of the response, and the presence of an unmet medical need [64].

Currently the data relevant to the assessment of immunogenicity for therapeutic biologics are dispersed throughout different locations of the eCTD including 2.7.4 Summary of Clinical Safety, 5.3.1.4 Reports on Biopharmaceutical Studies and 5.3.5 Reports of Efficacy and Safety Studies. The scattering of immunogenicity information in different modules of the regulatory file makes both the applicant's preparation of the immunogenicity information and the subsequent regulatory evaluation quite challenging. To facilitate both the clinical development of therapeutic biologics and the subsequent regulatory review process, both the EMA and FDA are now recommending a life-cycle management approach to immunogenicity through the creation of an ISI report (Summary of Immunogenicity Studies in EU). The FDA recommends that applicants begin populating the ISI early in product development and update at regular intervals as the individual product clinical program progresses through IND stages into the BLA and even post-approval stages. When used, this approach has made the reviewing of immunogenicity information easier and less time consuming. Therefore, in harmony with the revised EMA guidance on immunogenicity assessment of therapeutic proteins [63], FDA is considering including a section discussing recommendations for Integrated Summary Immunogenicity reports in the revised FDA draft guidance, currently scheduled for 2018.

\section{$P K, P D, A D A$ data correlation \& the merit of $N A b$ assays}

The 2017 White Paper in Bioanalysis introduced a scientific, assay agnostic approach for understanding the presence and impact of neutralizing antibodies (NAbs), highlighting the concept of 'biomarkers of NAbs' [18]. There is broad scientific agreement that there is a need to build an understanding of in vivo neutralizing activity, and regulators typically expect to see this determined through deployment of a standalone NAb assay. To date, this has been the standard approach, yielding two reportable results: positive for NAb or negative for $\mathrm{Nab}$ although sample results may in some cases be inconclusive due to drug tolerance issues. However, interpretation of reportable results is not always conclusive. While a positive NAb result is interpreted as presence of NAb, the clinical relevance is then determined by evaluating impact on safety, exposure and clinical efficacy. Negative NAb results present potential ambiguity from the outset, either indicating a genuine lack of NAb or the inability to detect NAb with the selected assay. Discriminating between these two possibilities to assess clinical relevance again requires evaluation of impact 
on safety, exposure and clinical efficacy. Therefore, in some cases, a standalone NAb assay may not produce data that offers clear insight into clinically meaningful in vivo neutralizing activity.

Consequently, it has been proposed that the decision to deploy a standalone NAb assay or other/biomarker assay that provides an integrated view of NAb impact should occur on a case by case basis. Some considerations when making this decision include the therapeutic molecule and its MOA, the risk assessment, the suite of assays available to interrogate its physiological impact and the quality of those assays. The assays that can best inform NAb impact should be deployed and emerging data leveraged to learn and refine strategy. An example of this integrated data approach was presented in a case study for a low risk $\mathrm{mAb}$ with antagonistic mechanism of action, where the risk assessment indicates that the key risk posed by NAb would be loss of exposure and potential impact on efficacy [65]. In this case study, binding ADA and exposure data generated from a free PK assay were monitored in an integrated fashion to inform on the presence of neutralizing activity and its relationship with clinical outcomes. Using this approach, in vivo neutralizing activity in $\mathrm{ADA}(+)$ subjects was interpreted as follows:

- $\mathrm{ADA}(+)$ with no impact on exposure levels or clinical efficacy is interpreted as non-neutralizing;

- $\mathrm{ADA}(+)$ with reduced exposure, but no impact on clinical efficacy, is interpreted as presence of neutralizing activity that is not clinically meaningful;

- $\mathrm{ADA}(+)$ with reduced exposure and reduction or loss of efficacy is interpreted as presence of clinically meaningful neutralizing activity. Loss-of-exposure neutralizing activity may result from the presence of neutralizing antibodies or clearing ADA response [60].

During the discussions, it was reiterated that approaches should be determined on a case by case basis. It was also discussed that in vivo translatability is complex and dynamic. It is dependent on a number of factors including the duration of the evaluation, patient population and the NAb assay format. Testing for NAb is a component of the characterization of the antibodies generated during an ADA response. However, the more distal the efficacy outcome is from what the assay is measuring, the more challenging it becomes to determine definitive correlations. Regulators again recommended early scientific engagement to align on the best path for any given therapeutic.

\section{Understanding clinical relevance of $A D A / N A b$}

Factors that determine the likelihood of success in accurately assessing ADA clinical impact include study design/clinical endpoints, MOA of the therapeutic, and the variety/characteristics of bioanalytical methods (immunogenicity, PK and biomarkers). A thorough understanding of the therapeutics' clinical pharmacology [66], coupled with an appropriate bioanalytical strategy maximizes the ability to generate data that can be analyzed to further understand the true clinical impact of ADA.

For scientists to establish an accurate immunogenicity profile for the drug in the treated patient population, several factors should be considered. During clinical development, clinical relevance of ADA may differ across sub-populations of patients (e.g., relative disease severity, concomitant medication) and therefore may need to be described within the context of each study [67]. In addition to determining the incidence, magnitude and persistence of response, the clinical impact of ADA upon re-exposure and on long-term use of the therapeutic must also be evaluated. This requires sufficient sampling (coinciding ADA and PK sampling is ideal) and an in-depth statistical analysis plan. Furthermore, when more broadly considering clinical relevance, it is important to remember that data sets generated during clinical development are limited and definitive conclusions are often only derived/refined later in the post-approval setting after more extensive and longer longitudinal data are analyzed.

When determining clinical impact, it is also important to go beyond simply reporting positive and negative [60]. While clinical development programs frequently provide data to describe ADA incidence, reporting of ADA titers is a regulatory expectation and available data suggest that these results are relevant and useful. Reporting of titers should include information - for example, neat titer or titer based on calculations of MRD (as well as how MRD was determined) followed by a statistical analysis to determine correlations with impact on exposure and clinical outcomes. This correlation analysis may be linear or non-linear. In this manner, an understanding of patients at a higher risk (safety or limited efficacy) can be developed and plans put in place to adequately inform risk-benefit treatment decisions. Lastly, as assay sensitivity improves, longitudinal studies correlating the ADA levels with clinical consequences may help determine the levels of ADA that are clinically meaningful. 


\section{Isotyping \& domain/ epitope mapping of ADA: utility of ADA isotyping assays?}

There are five types of antibody classes: IgA (two subclasses), IgD, IgE, IgG (four subclasses) and IgM, each with specific functionality. IgE isotyping information is sometimes desired because $\operatorname{IgE}$ is known to trigger histamine release from mast cells and basophils and is involved in anaphylaxis reactions. However, it has been challenging to correlate ADA IgE data with anaphylaxis events [68]. Generating data for ADA IgE isotyping at the outset does not offer any benefit nor does it always provide any predictive value. However, upon occurrence of an anaphylactic event, ADA IgE isotyping may help to explain the mechanism underlying the event in the patient. Once that information is understood for a given product, it may potentially be used to identify patients at risk so appropriate measures to mitigate this risk can be considered.

Using isotyping assays to characterize and understand the nature and dynamics of ADA responses is one of the last steps of the multi-tiered approach for assessing immunogenicity of a biotherapeutic. Isotyping is usually performed using a FFP approach and when necessary to answer specific questions.

Epitope mapping is very rarely requested. However, MDBs (e.g., ADC, fusion proteins, multi-specific mAbs having two or more different binding arms) may evoke immunogenicity responses, and hence require more detailed immunogenicity monitoring and testing strategies driven by a thorough immunogenicity risk assessment [69]. Domain mapping is often performed for multi-domain proteins at the confirmatory stage to characterize immune dominant regions [70]. This is typically accomplished using competitive binding assays but individual assays may also be used at the screening stage. Competitive binding, which is performed routinely to confirm the presence of antibodies, is a more streamlined approach requiring fewer resources, but can produce ambiguous results. Assays for individual domains may be more conclusive, although their development and validation are more demanding. If in doubt about whether isotyping or domain-specific assays are needed, sponsors should consult with the corresponding regulatory agencies.

\section{Post-marketing ADA monitoring: strategies, regulatory expectations \& clinically meaningful ADA assays}

Pre-licensing investigation of immunogenicity potential of new biotherapeutics is a regulatory expectation for product approval. Although registrational trials provide adequate information for approval, post marketing monitoring may be conducted to obtain a more complete and long-term understanding of immunogenicity and its impact. For post marketing monitoring, typically the same assay used in clinical development is employed to support Phase IV studies at a regulated bioanalytical laboratory. If the assay is made available to physicians interested in informing treatment decisions, the appropriate venue for conducting these assays would be a CLIA certified lab. In some instances, biologic drug license holders may perform immunogenicity testing for physician-investigators studying novel clinical indications for marketed products.

The foundation of a proper analysis of clinical significance of immunogenicity is an ADA assay that has adequate performance. Regulatory experience is that these assays often have insufficient sensitivity, leading to inadequate or even misleading information to the prescribers at the time of licensing. As a result, recent FDA and EMA guidance/guidelines [62,63] propose some higher sensitivity and improved drug tolerance goals for immunogenicity assays. Therefore, regulators do consider an assay's ability to detect in-study immunogenicity as an important indicator of the assay's true suitability above and beyond PC performance in validation.

ADA assay sensitivity is usually determined based on a positive control (either an anti-drug monoclonal antibody or a polyclonal antibody) and does not truly reflect the assay's ability to detect low affinity, polyclonal patient ADA responses. It is recommended that assays have sufficient sensitivity to detect ADA in the presence of the highest levels of drug expected to be present at the time of planned sampling. The sensitivity of the assay employed should be designed to provide clinically meaningful data that help correlate any impact of ADA on PK, PD, efficacy or safety. Low positive results from supersensitive assays $(<10 \mathrm{ng} / \mathrm{ml})$ should be interpreted cautiously and efforts made to distinguish signal from noise since they may not be true positives as the signal is in the range of assay noise, leading to inaccuracy in assessing clinical impact of ADA. Regulators point out that supersensitivity is not a problem if the sponsors stratify ADAs according to their titer (i.e., by assessing clinical impact based on titer range).

Measuring ADA titers, in addition to ADA status (positive versus negative), provides a means of distinguishing the magnitude of ADA responses and in many cases reveals that higher ADA titer has a greater impact on clinical outcomes - for example, decrease in drug exposure. However, demonstrating a quantitative correlation between absolute titers of ADA and the change in drug exposure has been challenging, and further investigation into comprehensive quantitative approaches is needed. Assay signal to noise $(\mathrm{S} / \mathrm{N})$ can be a valid alternative to titer for 
assessment of the magnitude of an ADA response under the appropriate circumstances and may generate equivalent results during analysis of impact of ADA on PK [71].

Despite recent advances in new and evolving technologies, antibody assays that meet current guidelines can sometimes be challenging to develop. There are no reference materials for antibody assays for most biotherapeutics (the exception is the first WHO EPO antibody reference panel [72]) making it difficult for laboratories involved in this work to assure the performance of their assays in the clinical setting [73]. With the emergence of biosimilars, the availability of a universal reference antibody/panel as positive controls to standardize ADA testing for different biotherapeutics would provide a consistent basis for ADA evaluation. Reference materials would also improve the confidence in the data, facilitate comparability of results and perhaps also allow clinical decision-making for the benefit of the patient. This was emphasized by clinicians and manufacturers at a workshop on immunogenicity assessment convened at the EMA [74].

On behalf of the WHO, NIBSC plans to make available either a reference antibody or an antibody panel as positive control(s) to standardize the testing for antibodies across different assay platforms where possible and different laboratories for a range of biotherapeutics. Provision of such positive controls through collaboration and active participation (e.g., clinicians, academia and industry) would also facilitate the immunogenicity assessment of biosimilar medicines and support access to safe and effective medicines [75].

\section{Cut points}

The detection, monitoring and characterization of ADA constitute critical steps in the development of protein therapeutics because of potential implications in both safety and efficacy of the drug. The CP of an immunogenicity assay serves as the threshold to define the subject as $\mathrm{ADA}$ positive or negative. The determination of appropriate assay cut points is a critical component in the assessment of immunogenicity during the drug development process. The presence of pre-existing drug-specific or cross-reactive antibodies in drug naïve individuals can complicate the determination of biologically relevant assay $\mathrm{CP}$ values and impact immunogenicity assessment.

\section{Issues in cut point determination \& $A D A$ assay drug tolerance \& sensitivity}

ADA assay cut points are usually determined by the analysis of a panel of individual samples, by different analysts, over different days, to account for both biological and analytical variations. Factors such as sample size, population used (normal versus disease), and statistical methods used can greatly influence the $\mathrm{CP}$ values obtained resulting in very different values. It was recommended to examine several methods to establish CP factors so that the appropriate $\mathrm{CP}$ is generated to distinguish real pre-dose positives from biological/analytical noise. The three types of cut points that can be employed were discussed in detail in Shankar et al. [76]. Typically, floating cut points are more appropriate for binding ADA assays [77]. Fixed cut points are usually not used for binding ADA assays, but are often appropriate for neutralizing ADA assays. Dynamic cut points in general should be avoided and it was advised that the assay be redeveloped instead of using dynamic cut points. Since biological variations generally exceed analytical variations, using larger numbers of baseline samples to account for the heterogeneity in the study population may generate more appropriate population cut points.

The current regulatory guidance recommends that screening and confirmation cut points be set at the upper $95^{\text {th }}$ and $99^{\text {th }}$ percentiles, respectively, to allow the presence of false positives and minimize the risk of false negative results [62]. The screening assay $\mathrm{CP}$ should correspond to a response that has low probability of occurring (e.g., $\mathrm{p}=0.05$ ) when samples from drug-naïve subjects are tested in the assay. The underlying assumption is that analytical variability of the assay is lower than the biological variability within the subject population or the longitudinal variability of a non-ADA positive untreated patient. The concept is that the $\mathrm{CP}$ will correspond to the lowest response that cannot be attributed to the normal assay variability given a certain critical probability (e.g., $\mathrm{p}=0.05$ or $\mathrm{p}=0.01$ ). Accordingly, the $\mathrm{CP}$ demarcates responses that can be expected to arise during routine operation of the assay from the responses that are unlikely to originate by chance and therefore are presumably biological in origin. Statistical procedures, particularly the outlier analysis, recommended for CP determination tend to reduce biological variability and may result in cut points that are very low and do not represent the entire population. It is expected that tiered testing should reduce the number of false positives; however, due to correlation of the screening and confirmatory responses, the tiered assays with low CP may generate a significant number of false positives [78]. It was agreed that it may be appropriate to evaluate setting up CP rules in the future to better differentiate the true positive samples, particularly for ultrasensitive assays. It was also agreed that some of the 
recommendations in current papers [76,79] might not really reflect what the industry is seeing when dealing with disease populations.

ADA assay sensitivity is related to the affinity of the surrogate PC antibodies used in the assay. Regulators' expectations regarding drug tolerance for ADA assays are that the assay be sensitive to ADA with the levels of drug expected at the time of sampling. For drugs with a short half-life, scientists should aim for $\mathrm{C}_{\text {trough }}$. For those with a long half-life, the highest drug concentrations that will be present at the times sampled should be targeted. Due to limitations in assay technology, target interference, product, matrix and/or posology, it is not always possible to develop an assay with adequate sensitivity/drug tolerance. Furthermore, it can be difficult to get a patient PC that adequately reflects the diversity of the clinical samples, for use as a check of the assay performance. Regulators indicated that sponsors are usually encouraged to develop PC specific for their product. It should be noted that long-term stability of the PC is not necessary for ADA assays and there is no requirement for ISS. When a commercial PC is used, then a CoA, detailing amongst other things the lot number and the expiry date of the product is needed.

In the 2017 White Paper in Bioanalysis [18], regulators indicated they are looking for a $1 \%$ failure rate for the LPC to demonstrate a consistent ability of the assay to detect low ADA concentrations. Typically, the LPC should be statistically determined, but there may be times, such as when using ultrasensitive assays, where another approach can be justified (e.g., two-to three-fold signal to noise ratio). Within the same population, it is unusual to have different CP, whereas for different diseases or disease states, different CP may occur. Age, gender and ethnicity may also impact $\mathrm{CP}$ and the applicability of the original $\mathrm{CP}$ needs to be confirmed. When dealing with markedly different CP values for different disease populations or different studies, a LPC concentration that covers different populations is sometimes preferred, but may not always be suitable.

Despite the limitations outlined above, "supersensitive ADA assays" which have a defined sensitivity of $<10 \mathrm{ng} / \mathrm{ml}$ are now being used. Their use has raised the question of the relevance of detecting ADAs at very low levels and recommending for a 5\% FPR as opposed to flexibility in the guidance/guideline. Provided these assays are performing inside the optimal range for the platform, very low levels of $\operatorname{IgG}$ are likely not clinically relevant. The target is $5 \%$ FPR, but the actual acceptable range detected is $2-11 \%$ [77]. However, in the assessment of $\operatorname{IgE}$ antibodies possibly linked to hypersensitivity, the required sensitivity may be different due to their intrinsically lower physiological concentration, short half life, as well as the difficulty in obtaining samples at peak IgE concentrations in the blood.

The recommendation in the 2017 White Paper in Bioanalysis [18] seemed to indicate that drug tolerance has become more important than sensitivity. These are not independent factors and a balance needs to be achieved. Indeed, regulators recommend that the assay validation establish the sensitivity of the assay in the context of the likely drug concentration at the time of sampling. Various strategies are used to increase drug tolerance. The use of tools such as acid dissociation may reduce the stability of the ADA; thus, a clear benefit of the employed methods on ADA detection should be demonstrated. When choosing a NAb assay format where a cell-based assay would be preferred but suffers from poor drug tolerance, regulators have shown a willingness to accept ligand binding NAb assays if they are more sensitive, have better drug tolerance and are deemed appropriate based upon the MOA of the therapeutic [85]. Consultation with regulators to achieve scientific alignment is encouraged.

\section{Issues with dynamic, NAb, biosimilar cut points \& outliers}

Current practice of CP determination employs analytical/biological outlier removal and distributional assumptions to assure a minimum specific FPR with high confidence. The statistical approach used regarding the removal of outliers, especially when dealing with heterogeneous disease populations, can affect the FPR observed for the therapeutic being tested. The ability to set an appropriate $\mathrm{CP}$ is dependent on appropriately differentiating true biological positive samples and analytical outliers. In populations with relatively few outliers, a distinction between analytical and biological outliers may not be necessary and can be handled through outlier exclusion using standard approaches such as box plots [76]. Assays where the CP is close to the technical limit of the instrumentation are particularly challenging. In such cases, the widely used standard symmetrical box plot method can result in a significantly higher false positive rate than the expected 5\% for clinical screening assays. In addition, the identification and removal of outliers becomes more complicated when there is pre-existing reactivity in a large fraction of the population tested. For vaccine based assays, presence of pre-existing seroreactivity can impact the seroresponse/seroconversion criterion. The measure of a positive response to vaccines relies on the assay used, the epidemiology of the population being considered as serostatus and consequent protection can vary based on 
previous exposures. Additionally, for some vaccines like human papilloma virus (HPV), multiple panels of serum with negative to varying levels of positive seroreactivity are needed to establish a relevant cut off [80]. There is no industry standard for setting up CP and data reporting in presence of pre-existing reactivity, but there are multiple approaches being used to select the most appropriate $\mathrm{CP}$ [81].

Comparisons among pre- and in-study populations are frequently made to assess the need for SSCP. Guidance states that the CP should be confirmed to be appropriate. After evaluation of the FPR of the baseline samples of the instudy population and comparison of means and variances of the pre- and in-study populations, a biostatistician may be consulted to determine if pre-study or study-specific CP should be used. To determine the SSCP for monoclonal antibodies using a small sample size with few or no repeats, one approach discussed was Rosner's multiple-outlier test for outlier identification. Also used were non-normal Bayesian hierarchical models (e.g., Johnson SU or skew-t) to separate biological and analytical variation, and then estimate the $\mathrm{CP}$ as a conservative low bound on a quantile of the posterior predictive distribution of future signals [78,82]. Although statistical methods for cut-point calculations have been discussed in several statistical and white papers in the last decade, less pronounced emphasis has been given to outlier detection approaches as a key component of cut-point determination. It was re-confirmed in the 2017 White Paper recommendation [18], that the use of an outlier factor of 1.5 might not consider the variability observed in some disease populations and may lead to the removal of a high number of outliers, resulting in lower $\mathrm{CP}$ values. As such, higher outlier factors may be necessary to achieve an appropriate $\mathrm{CP}$ value.

\section{Recommendations}

Below is a summary of the recommendations made during the $12^{\text {th }}$ WRIB.

\section{Gene therapy}

1. For each GT modality, an assessment of bioanalytical challenges and strategies should be outlined and appropriate for each phase of drug development. Factors which must be considered for viral vector GT treatment modalities should include:

- Pre-existing immunogenicity and anti-capsid NAb;

- Development of humoral and cellular immune responses to both the vector and transgene;

- Strategies for the evaluation of PK and PD responses;

- Risk of viral shedding and environmental exposure.

2. When biodistribution studies are difficult to perform due to tissue biopsy limitations, non-clinical studies can provide biodistribution information. However, the translatability of this information may depend on the vector or mode of delivery of the transgene or the transgene itself:

○ Exposure and biodistribution are typically determined by qPCR (or equivalent assay), with normalization to an appropriate host reference sequence;

- Expression of the transgene (PD) may be measured by RT-PCR, or by detection of expressed protein by LBA or LCMS.

3. Vector shedding is critical to perform with viral-based vectors to minimize the risk of disseminating infectious material. Shedding levels should be characterized:

o If present, the potential infectivity of the shed virus is determined by a cell-based infectivity assay. A surrogate viral protein may be used to monitor the infectious titer present by staining for a late stage viral replication protein, in a cell based assay. Cell lysates and fixed cells may also be considered, in-lieu of an infectious particle assay.

4. qPCR assays are a standard technique for regulated bioanalysis in the gene therapy space:

$\circ$ qPCR validation assay parameters:

- Similar to those for LCMS and LBA validations;

- NTC should be negative;

- Primers should be specific for the vector and transgene;

- Sensitivity can be established based on the number of copies detected per microgram of genomic DNA. Sensitivity requirements may be different in preclinical vs. clinical assays;

- Assay performance controls and QCs should be established to monitor assay performance.

5. Cellular immunity should be characterized. For the evaluation of cellular immunity, ELISPOT is the gold standard, although other methods based on flow are being investigated. Similar processing and storage of PBMC samples at each site is critical in order that the functional assay not be compromised: 
- When deciding what should be evaluated for cellular immunity, capsid and gene products were all considered important;

- Anti-capsid NAb (TI) testing should be considered as an enrollment criterion.

\section{Critical reagents}

6. A comprehensive and robust program to handle critical reagents should include SOPs and work instructions for preparation, qualification, labeling and storage. Proper documentation of reagent characterization should be available;

7. Defining the critical attributes of required reagents and having a testing strategy for characterization of key attribute determinants is highly recommended;

8. An electronic inventory system is ideal to track inventory and expiry of all reagents;

9. Characterization of critical reagents should include identity, concentration, binding activity, purity and labeling efficiency, and freeze-thaw stability. Orthogonal characterization based on physical attributes might be recommended depending on the reagent type and use;

10. Several aliquots of key reagents should be retained to ensure bridging experiments and troubleshooting efforts can be performed when necessary. Forced degradation studies are not recommended for critical reagents;

11. For reagents prone to aggregation, SEC-based characterization may be required. The addition of sucrose or trehalose may help to minimize the impact of freeze-thaw on aggregation, but no universal formula has been identified.

\section{Biomarker assay validation}

12. Important: a concise regulatory guidance/guideline for BAV is desired by many, rather than the industry relying on best practices;

13. COU must drive all aspects of patient biomarker testing;

14. Developers should consult the regulators early in the development program to ensure the proper testing strategy is implemented, especially if the biomarker is a clinical endpoint. Current consensus in the C-Path document points scientists to follow the FNIH Biomarkers Consortium Framework for Evidentiary Standards for Biomarker Qualification;

15. Important: it is critical to distinguish between the predictive biomarkers that are eventually targeted to be applied for decision making at the patient level and the biomarkers utilized only for internal decision making by drug developers:

- Biomarker qualification is a drug or product independent determination by the FDA, and once established for a specific COU, it can be used without re-review for other drug products across multiple drug development programs for the same COU;

- Only a tiny fraction of biomarkers will be submitted for qualification.

16. If a biomarker is used for regulatory decisions such as patient selection, the assay must be adequately validated for its specific COU:

- However, it is crucial that the biomarker community move away from applying the BMV guidance to BAV in a prescriptive or overly rigid fashion.

17. Biomarkers of interest that are not intended for regulatory decision making should use FFP BAV:

- FFP BAV recommendations presented in the 2015-2017 White Papers in Bioanalysis $[12,15,18]$ are still valid.

18. Bridging studies should be considered when there are assay changes within or between studies, including sample type, sample collection times, using multiple versions of an assay, and/or different lots of commercial kits;

19. The approach to assessing biological variability of a biomarker in the drug development realm would be to, first, generate a hypothesis, validate the assay, gather the data and then interpret the data in collaboration with the statisticians. Consider the COU, select an appropriate number of relevant subject samples, and apply an appropriately validated assay to evaluate the samples to establish biological variability.

\section{Flow cytometry}

20. The flow cytometry approach that best fits the method and COU should be used (direct and indirect are both viable options); 
21. A FFP approach will need to guide the specific validation parameters assessed for flow cytometry-based biomarkers representing exploratory, pharmacodynamic and predictive endpoints:

- FFP flow cytometry validation parameters typically should focus on specificity, intra- and inter-batch precision, and sample stability, and, when appropriate, interferences, reference ranges, and controls;

- Ensure proper sample handling and temperature controls at the collection sites, thereby preserving sample stability;

- Establish QC process and lab comparability for multi-lab sample analysis that occurs for longer durations of sample testing;

- Critical reagents (e.g., antibodies, permeabilization buffers) should be characterized during development and validation, and properly controlled;

- During development of flow cytometry methods for use in CAR T-cell therapy, obtain an appropriate sample, generated from healthy donors. Furthermore, custom reagents are needed with appropriate life cycle management plans;

- An FFP approach for run acceptance criteria based on critical assay parameters or sample acceptance criteria should be used. These criteria may include the number of minimum events collected, RBC lysis, and established QC criteria (if using);

- Real time sample analysis is scientifically ideal but does require method validation before study start;

- To minimize the impact of challenges with the manipulation of PBMC preparations and cryopreservation, it is recommended to implement rigorous training at the collection site and monitor the quality of the samples;

- Accuracy is not an appropriate parameter in flow cytometry validations.

22. Gating strategy and template is a critical part of the assay:

- Rules-based decision making on gating, defined in validation, may be optimized in-study;

- Quality control review of the gating is required and any changes should be properly documented;

- Use rules-based gating when determining the cut-off of FMO for poorly resolved population.

23. Whole blood flow cytometry is recommended for TE biomarkers because it limits manipulation of the sample and allows for the most accurate assessment of target engagement:

- Validation of TE biomarkers should be FFP based on the COU;

- Operator training and multi-site qualification of TE biomarkers using flow cytometry should include precision, but sample stability is not required;

- Parameters around biological variability (both cross sectional and longitudinal) and potential interferences should be established and accounted for in the validation of TE biomarkers using flow cytometry.

24. Flow cytometry is not generally recommended for tissue $\mathbf{R O}$ due to technical challenges that are likely to impact the integrity of the target and the dissociation of the drug. If this avenue is pursued, the described challenges should be thoroughly assessed during method development and evaluated for feasibility of implementation:

- The target needs to be detectable; ideally a RO window with sufficient dynamic range should be established during development;

$\circ$ The detection sensitivity of the measurements (PD effect) has to be greater than the assay/biological variability;

- Establish 100\% RO (maximum inhibition) by spiking in excess drug.

\section{ADA Assays \& PK, PD, ADA data correlation}

25. An understanding of in vivo neutralizing activity of ADAs is required, and regulators typically expect to see a standalone NAb assay used as part of this assessment:

- An integrated data approach was discussed, using a case example, where $\mathrm{ADA}(+)$ and exposure data from a free PK assay are monitored in an integrated fashion to inform presence of in vivo neutralizing activity and the relationship with clinical outcomes. The decision to deploy a standalone NAb assay or other/biomarker assay of in vivo NAb impact should be evaluated on a case by case basis. Regulators encourage scientific engagement early on to align on the best path for any given therapeutic.

26. It was recommended that a risk-based approach for the immunogenicity risk assessment for IND support, which is also advocated in the immunogenicity guidance from EMA [63], is needed to balance the potential harm with potential benefits of a new biotherapeutic throughout clinical development. This assessment 
should be based on the likelihood of developing an immune response, the risk of a response to the patient, the reversibility of the response, and the presence of an unmet medical need;

27. Both the FDA and the EMA recommend that the sponsors submit an Integrated Summary of Immunogenicity/Summary of the Immunogenicity Program that will justify the chosen risk-based approach and facilitate the regulatory review. The FDA is recommending a life-cycle management approach to immunogenicity through the creation of an Integrated Summary of Immunogenicity report that applicants begin populating early in product development, and update at regular intervals as the individual product clinical program progresses through IND stages into the BLA and even post-approval stages;

28. A thorough understanding of the therapeutic's pharmacology, coupled with an appropriate bioanalytical strategy (ideally using assays that test specific hypotheses regarding clinical impact) maximizes the ability to further understand the true clinical impact of ADA;

29. Clinical relevance may differ across sub-populations of patients (e.g., relative disease severity, concomitant medication) and therefore may need to be described within the context of each study:

- It is important to determine the incidence, magnitude and persistence of the response, as well as the impact of re-exposure and on long-term use of the therapeutic. This requires sufficient sampling (coinciding ADA and PK sampling is ideal) and an in-depth statistical analysis;

- Reporting of titers should include relevant information - for example, neat titer or titer based on calculations of MRD (as well as how MRD was determined) followed by statistical analysis to determine correlations with impact on exposure and clinical outcomes;

- Data sets generated during clinical development are limited and such conclusions may need to be reassessed in the post-market phase;

- When determining clinical impact analyses, reporting beyond the incidence of positive responses is required. The persistence of the immune response, as well as the magnitude of the response (titers) may provide critical information to determine if an ADA response is clinically relevant;

- Pharmacokinetic measurements may be useful in evaluating clinical relevance of the ADA.

30. In most cases, generating data for ADA IgE isotyping at the outset does not offer any benefit nor does it provide any predictive value. However, upon occurrence of a hypersensitivity reaction event, IgE isotyping may help to explain the mechanism underlying the event in the patient. Once understood, it may be used to predict future events:

○ Isotyping, including IgE, should only be performed using a fit-for-purpose approach and when necessary to answer specific questions. Regulatory requests for isotyping are often driven by safety or efficacy concerns, and not routinely required.

31. Domain mapping is recommended for multi-domain complex proteins (e.g., ADC, fusion proteins, bispecific Abs) at the confirmatory stage to characterize immune dominant regions;

32. Post marketing monitoring may provide a more complete understanding of the impact of relative immunogenicity. For post marketing monitoring, typically the assay used in clinical development is employed to support Phase IV studies at a regulated bioanalytical laboratory:

- If the assay is made available to physicians interested in informing treatment management decisions, the appropriate venue for conducting these assays would be a CLIA certified lab;

- In specific regulatory instances, license holders may perform immunogenicity testing for physicianinvestigators studying novel clinical indications for marketed products.

33. Regulators consider an assay's ability to detect in-study immunogenicity as an important indicator of the assay's suitability above and beyond PC performance in validation.

Cut points

34. Assay (analytical) variability and sample (biological) variability are both important when determining CP:

- Examine the most suitable ways to determine CP factors so that the appropriate $\mathrm{CP}$ is generated to distinguish real positives from biological/analytical noise;

- The screening assay CP should incorporate a false positive rate (e.g., $\mathrm{p}=0.05)$ when samples from drug-naïve subjects are tested in the assay;

- It may be appropriate to evaluate setting up CP rules in the future to improve identification of positive samples;

- When determining CPs, the target FPR is 5\%, but the actual range that may be obtained is $2-11 \%$. 
35. ADA assay sensitivity is related to the affinity of the surrogate $P C$ antibodies used in the assay:

- It is generally justifiable not to include long-term stability for the PC for ADA assays;

- 'Supersensitive ADA assays' which have a defined sensitivity of $<10 \mathrm{ng} / \mathrm{ml}$ are now often being used but are not required. Assays should be sensitive enough to detect clinically relevant ADA;

○ When dealing with different CP values for different disease populations or different studies (e.g., different age group, ethnicity, etc.), the adequacy of the LPC concentration should be confirmed. Sometimes an LPC that covers different populations instead of just one may be selected.

36. Regulators' expectation regarding drug tolerance for ADA assay depends on the half-life of the therapeutic and how it is administered - that is, the expected drug concentration in the clinical samples:

- For drugs with a short half-life, scientists should aim for $\mathrm{C}_{\text {trough }}$. For those with a long half-life, the highest drug concentrations that will be present at the times sampled should be targeted;

- Increasing drug tolerance should only be done to gain sufficient sensitivity to detect ADA in clinical samples. A balance between sensitivity and drug tolerance is needed.

37. When choosing between a cell-based NAb assay with poor drug tolerance and a LBA with better drug tolerance, regulators have shown a willingness to accept ligand binding NAb assays that are more sensitive and have better drug tolerance provided they are biologically relevant. If ligand binding NAb assays are not appropriate based on the MOA of the therapeutic, then cell-based assays should be used. Consultation with regulators to provide justification is encouraged;

38. Fixed cut points can be used when assay runs demonstrate similar means and variances. Floating cut points should be used when means vary between relevant factors (e.g., analysts, plates or assay runs), but variances around the mean remain similar. In general, floating cut points are typically the best choice for long-term assay robustness and consistent performance:

$\circ$ The use of dynamic cut points is not recommended, assay redevelopment and/or additional training should be considered.

39. Comparisons among pre- and in-study populations (e.g., FPR) are frequently made to assess the need for SSCP. Guidance states that the CP should be confirmed. If there is concern that the pre-established CP is not appropriate, then a biostatistician should be consulted to determine if the two populations are statistically different. If not, the pre-study CP should be used;

40. For vaccine specific immunogenicity assays, the regulatory guidance around serostatus cut-offs and preexisting seroreactivity need more clarity. Due to complexity of vaccine antigens and their cross reactivity to other pathogens, there is a lack of adequate reagents and reference standards that can support interpretation of seroconversion and seroreactivity.

Acknowledgements

- The US FDA, Europe EMA, UK MHRA, Brazil ANVISA, Health Canada, Japan MHLW and Finland Fimea for supporting this workshop.

- C Beaver (Syneos Health), S (Julia) Cho (US FDA), J Kamerud (Pfizer), S Keller (AbbVie), F Garofolo (Angelini Pharma), J Marini (Janssen), A Musuku (Pharmascience), H Neubert (Pfizer), T Olah (Bristol-Myers Squibb), R Pillutla (Bristol-Myers Squibb), S Richards (Sanofi), L Stevenson (Biogen), A Torri (Regeneron), S Vinter (UK MHRA), J Welink (EU EMA), Y Xu (Alnylam) and E Yang (GlaxoSmithKline); for chairing the workshop and/or the white paper discussions.

- The Biogen Team (L Stevenson, C Stebbins, S Purushothama and D Mehta) for the pre-review of Part 3.

- All the workshop attendees and members of the Global Bioanalytical Community who have sent comments and suggestions to the workshop to complete this White Paper.

- Future Science Group as a trusted partner.

\section{References}

1 Savoie N, Booth BP, Bradley T et al. The 2nd Calibration and Validation Group Workshop on recent issues in Good Laboratory Practice bioanalysis. Bioanalysis 1(1), 19-30 (2009).

2 Savoie N, Garofolo F, van Amsterdam P et al. 2009 White Paper on recent issues in regulated bioanalysis from the 3rd Calibration and Validation Group Workshop. Bioanalysis 2(1), 53-68 (2010).

3 Savoie N, Garofolo F, van Amsterdam P et al. 2010 White Paper on recent issues in regulated bioanalysis and global harmonization of bioanalytical guidance. Bioanalysis 2(12), 1945-1960 (2010). 
4 Garofolo F, Rocci M, Dumont I et al. 2011 White Paper on recent issues in bioanalysis and regulatory findings from audits and inspections. Bioanalysis 3(18), 2081-2096 (2011).

5 DeSilva B, Garofolo F, Rocci M et al. 2012 White Paper on recent issues in bioanalysis and alignment of multiple guidelines. Bioanalysis 4(18), 2213-2226 (2012).

6 Stevenson L, Rocci M, Garofolo F et al. 2013 White Paper on recent issues in bioanalysis: "hybrid" - the best of LBA \& LC/MS. Bioanalysis 5(23), 2903-2918 (2013).

7 Fluhler E, Hayes R, Garofolo F et al. 2014 White Paper on recent issues in bioanalysis: a full immersion in bioanalysis (Part 1 - small molecules by LCMS). Bioanalysis 6(22), 3039-3049 (2014).

8 Dufield D, Neubert H, Garofolo F et al. 2014 White Paper on recent issues in bioanalysis: a full immersion in bioanalysis (Part 2 hybrid LBA/LCMS, ELN \& regulatory agencies' input). Bioanalysis 6(23), 3237-3249 (2014).

9 Stevenson L, Amaravadi L, Myler H et al. 2014 White Paper on recent issues in bioanalysis: a full immersion in bioanalysis (Part 3 - LBA and immunogenicity). Bioanalysis 6(24), 3355-3368 (2014).

10 Welink J, Fluhler E, Hughes N et al. 2015 White Paper on recent issues in bioanalysis: focus on new technologies and biomarkers (Part 1 - small molecules by LCMS). Bioanalysis 7(22), 2913-2925 (2015).

11 Ackermann B, Neubert H, Hughes N et al. 2015 White Paper on recent issues in bioanalysis: focus on new technologies and biomarkers (Part 2 - hybrid LBA/LCMS and input from regulatory agencies). Bioanalysis 7(23), 3019-3034 (2015).

12 Amaravadi L, Song A, Myler H et al. 2015 White Paper on recent issues in bioanalysis: focus on new technologies and biomarkers (Part 3 - LBA, biomarkers and immunogenicity). Bioanalysis 7(24), 3107-3124 (2015).

13 Yang E, Welink J, Cape S et al. 2016 White Paper on recent issues in bioanalysis: focus on biomarker assay validation (BAV) (Part 1 small molecules, peptides and small molecule biomarkers by LCMS). Bioanalysis 8(22), 2363-2378 (2016).

14 Song A, Lee A, Garofolo F et al. 2016 White Paper on recent issues in bioanalysis: focus on biomarker assay validation (BAV): (Part 2 hybrid LBA/LCMS and input from regulatory agencies). Bioanalysis 8(23), 2457-2474 (2016).

15 Richards S, Amaravadi L, Pillutla R et al. 2016 White Paper on recent issues in bioanalysis: focus on biomarker assay validation (BAV): (Part 3 - LBA, biomarkers and immunogenicity). Bioanalysis 8(23), 2475-2496 (2016).

16 Welink J, Yang E, Hughes N et al. 2017 White Paper on recent issues in bioanalysis: aren't BMV guidance/guidelines 'scientific'? (Part 1 - LCMS: small molecules, peptides and small molecule biomarkers). Bioanalysis 9(22), 1807-1825 (2017).

17 Neubert H, Song A, Lee A et al. 2017 White Paper on recent issues in bioanalysis: rise of hybrid LBA/LCMS immunogenicity assays (Part 2: hybrid LBA/LCMS biotherapeutics, biomarkers \& immunogenicity assays and regulatory agencies' inputs). Bioanalysis 9(23), 1895-1912 (2017).

18 Gupta S, Richards S, Amaravadi L et al. 2017 White Paper on recent issues in bioanalysis: a global perspective on immunogenicity guidelines \& biomarker assay performance (Part 3 - LBA: immunogenicity, biomarkers and PK assays). Bioanalysis 9(24), 1967-1996 (2017).

19 Lee JW, Devanarayan V, Barrett YC et al. Fit-for-purpose method development and validation for successful biomarker measurement. Pharm. Res. 23(2), 312-328 (2006).

20 Reflection paper on quality, non-clinical and clinical issues related to the development of recombinant adeno-associated viral vectors. European Medicines Agency, Committee for the Medicinal Products for Human Use (CHMP), London, UK (2010). http://www.ema.europa.eu/docs/en_GB/document_library/Scientific_guideline/2010/07/WC500094345.pdf

21 Guidance for Industry: Recommendations for Microbial Vectors used for Gene Therapy. US Department of Health and Human Services US FDA, Center for Biologics Evaluation and Research, Rockville, MD, USA (2016).

22 Guidance for Industry: Design and Analysis of Shedding Studies for Virus or Bacteria-Based Gene Therapy and Oncolytic Products Guidance for Industry. US Department of Health and Human Services US FDA, Center for Biologics Evaluation and Research, Rockville, MD, USA (2015).

23 Guidance for Industry: Preclinical Assessment of Investigational Cellular and Gene Therapy Products. US Department of Health and Human Services US FDA, Center for Biologics Evaluation and Research, RJockville, MD, USA (2013).

24 Guideline on the Non-Clinical Studies Required Before First Clinical Use of Gene Therapy Medicinal Products. EMA, Committee for the Medicinal Products for Human Use (CHMP), London, UK (2008).

25 Considerations for the Design of Early-Phase Clinical Trials of Cellular and Gene Therapy Products Guidance for Industry. US Department of Health and Human Services US FDA, Center for Biologics Evaluation and Research, Silver Spring, MD, USA (2015).

26 Gene Therapy Clinical Trials - Observing Subjects for Delayed Adverse Events. US Department of Health and Human Services US FDA, Center for Biologics Evaluation and Research, Rockville, MD, USA (2006).

27 Guideline on Follow-Up of Patients Administered with Gene Therapy Medicinal Products. EMA, Committee for Medicinal Products for Human Use (CHPM), London, UK (2009).

28 Boutin S, Monteilhet V, Veron P et al. Prevalence of serum IgG and neutralizing factors against adeno-associated virus (AAV) types 1, 2 , 5, 6, 8, and 9 in the healthy population: implications for gene therapy using AAV vectors. Hum. Gene Ther. 21(6), 704-12 (2010). 
29 Manno CS, Pierce GF, Arruda VR et al. Successful transduction of liver in hemophilia by AAV-Factor IX and limitations imposed by the host immune response. Nat. Med. 12(3), 342-7 (2006).

30 Schenk-Braat EA, van Mierlo MM, Wagemaker G et al. An inventory of shedding data from clinical gene therapy trials. J. Gene Med. 9(10), 910-21 (2007).

31 Mingozzi F, High KA. Immune responses to AAV vectors: overcoming barriers to successful gene therapy. Blood 122(1), 23-36 (2013).

32 Ewer KJ, Lambe T, Rollier CS et al. Viral vectors as vaccine platforms: from immunogenicity to impact. Curr. Opin. Immunol. 41, 47-54 (2016).

33 ICH Considerations: General principals to address virus and vector shedding. EMA, London, UK (2009).

34 Zen Z, Espinoza Y, Bleu T, Sommer JM, Wright JF. Infectious titer assay for adeno-associated virus aectors with sensitivity sufficient to cetect single infectious events. Hum. Gene Ther. 15(7), 709-715 (2004).

35 Geist BJ, Clements Egan A, Yang T-Y, Dong Y, Shankar G. Characterization of critical reagents in ligand binding assays: enabling robust bioanalytical methods and lifecycle management. Bioanalysis 5(2), 227-244 (2013).

36 Nicholson R, Lowes S, Cruz Caturla M et al. Conference Report: 6th GCC focus on LBA: critical reagents, positive controls and reference standards; specificity for endogenous compounds; biomarkers; biosimilars. Bioanalysis 4(19), 2335-2342 (2012).

37 Tatarewicz S, Miller JM, Swanson SJ, Moxness MS. Rheumatoid factor interference in immunogenicity assays for human monoclonal antibody therapeutics. Immunol. Methods 357(1-2), 10-16 (2010).

38 Kubiak RJ, Lee N, Zhu Y et al. Storage conditions of conjugated reagents can impact results of immunogenicity assays. J. Immunol. Res. 2016, Article ID 1485615 (2016).

39 Arnold M, Booth B, King L et al. Workshop Rreport: Crystal City VI - bioanalytical method validation for biomarkers. AAPS J. 18(6), 1366-1372 (2016).

40 Duke-Margolis Center for Health Policy. Public workshop: scientific and regulatory considerations for the analytical validation of assays used in the qualification of biomarkers in biological matrices.

https://healthpolicy.duke.edu/events/public-workshop-scientific-and-regulatory-considerations-analytical-validation-assays-used

41 Piccoli SP, Sauer JM. Points to consider document: scientific and regulatory considerations for the analytical validation of assays used in the qualification of biomarkers in biological matrices biomarker assay. Collaborative Evidentiary Considerations Writing Group, Critical Path Institute (C-Path) (2017). https://healthpolicy.duke.edu/sites/default/files/atoms/files/cpath_ptc_biomarker_qualification_assays_20170623.pdf

42 FNIH Framework for Defining Evidentiary Criteria for Biomarker Qualification. Biomarkers Consortium Evidentiary Standards Writing Group 2016. https:

//fnih.org/sites/default/files/final/pdf/Evidentiary\%20Criteria\%20Framework\%20Final\%20Version\%20Oct\%2020\%202016.pdf

43 FDA-NIH Biomarker Working Group. BEST (Biomarkers, EndpointS, and other Tools) Resource. Silver Spring (MD, USA), Food and Drug Administration (MD, USA), Bethesda (MD, USA), National Institutes of Health (MD, USA) (2016).

44 Cowan KJ, Amaravadi L, Cameron MJ et al. Recommendations for selection and characterization of protein biomarker assay calibrator material. AAPS J. 19(6), 1550-1563 (2017).

45 US Department of Health and Human Services, US FDA, Center for Drug Evaluation and Research, Center for Veterinary Medicine. Guidance for Industry, Bioanalytical Method Validation. Rockville, MD, USA (2018).

46 Nowatzke W, Cole TG, Bowsher RR. Systematic analytical validation of commercial kits for the determination of novel biomarkers for clinical drug development. Bioanalysis 2(2), 237-247 (2010).

47 Khan MU, Bowsher RR, Cameron M et al. Recommendations for adaptation and validation of commercial kits for biomarker quantification in drug development. Bioanalysis 7(2), 229-242 (2015).

48 Maecker HT, McCoy JP, Nussenblatt R. Standardizing immunophenotyping for the Human Immunology Project. Nat. Rev. Immunol. 12(3), 191-200 (2012).

49 Wood B, Jevremovic D, Bene MC et al. and Group IIW. Validation of cell-based fluorescence assays: practice guidelines from the ICSH and ICCS - part V - assay performance criteria. Cytometry B Clin. Cytom. 84(5), 315-23 (2013).

50 Hoffman RA, Wang L, Bigos M, Nolan JP. NIST/ISAC standardization study: variability in assignment of intensity values to fluorescence standard beads and in cross calibration of standard beads to hard dyed beads. Cytometry A 81, 785-96 (2012).

51 O'Hara DM, Xu Y, Liang Z et al. Recommendations for the validation of flow cytometric testing during drug development: II assays. J. Immunol. Methods 363(2), 120-34 (2011).

52 Wang Q-S, Wang Y, Hai-yan L et al. Treatment of CD33-directed chimeric antigen receptor-modified T cells in one patient with relapsed and refractory acute myeloid leukemia. Mol. Ther. 23(1), 184-191 (2015).

53 Kohrt HE, Tumeh PC, Benson D et al. Immunodynamics: a cancer immunotherapy trials network review of immune monitoring in immuno-oncology clinical trials. J. Immunother. Cancer 4(5), (2016).

54 Mueller KT, Maude SL, Porter Dl et al. Cellular kinetics of CTL019 in relapsed/refractory B-cell acute lymphoblastic leukemia and chronic lymphocytic leukemia. Blood 130(21), 2317-2325 (2017). 
55 Litwin V, Stewart J, Green C. Receptor occupancy by flow cytometry. Cytometry B Clin Cytom. 90B, 108-9 (2016).

56 Stewart JJ, Green CL, Jones N et al. Overview of flow cytometry receptor occupancy assays and their utility in drug development. Cytometry B Clin. Cytom. 90B, 110-6 (2016).

57 Green CL, Stewart JJ, Högerkorp CM et al. Recommendations for the development and validation of flow cytometry-based receptor occupancy assays. Cytometry B Clin. Cytom. 90B, 14-9 (2016).

58 Hougton R, Gouty D, Allinson J et al. Recommendations on biomarker bioanalytical method validation by GCC. Bioanalysis 4(20), 2439-2446 (2012).

59 Shankar G, Arkin S, Devanarayan V et al. The quintessence of immunogenicity reporting for biotherapeutics. Nat. Biotechnol. 33(4), 334-336 (2015).

60 Shankar G, Arkin S, Cocea L et al. Assessment and reporting of the clinical immunogenicity of therapeutic proteins and peptides harmonized terminology and tactical recommendations. AAPS J. 16(4), 658-673 (2014).

61 Guidance for Industry, Immunogenicity Assessment for Therapeutic Protein Products. US Department of Health and Human Services US FDA, Center for Drug Evaluation and Research, Center for Biologics Evaluation and Research, Rockville, MD, USA (2014).

62 Draft guidance for Industry, Assay Development and Validation for Immunogenicity Testing of Therapeutic Protein Products. US Department of Health and Human Services US FDA, Center for Drug Evaluation and GResearch, Center for Biologics Evaluation and Research Center for Devices and Radiological Health, Rockville, MD, USA (2016).

63 Guideline on Immunogenicity Assessment of Therapeutic Proteins. EMA, Committee for Medicinal Products for Human Use (CHMP), London, UK (2017).

64 Mytych DT, Hock MB, Kroenke M et al. A proposal to redefine clinical immunogenicity assessment. AAPS J. 19(3), 599-602 (2017).

65 Stevenson LF. Biomarkers, PK and immunogenicity: are we ready for integration? Bioanalysis 8(22), 2287-2289 (2016).

66 Chirmule N, Jawa V, Meibohm B. Immunogenicity to therapeutic proteins: impact on PK/PD and efficacy. AAPS J. 14(2), 296-302 (2012)

67 Vennegoor A, Rispens T, Strijbis EM et al. Clinical relevance of serum natalizumab concentration and anti-natalizumab antibodies in multiple sclerosis. Mult. Scler. 19(5), 593-600 (2013).

68 Baker DL, Nakamura GR, Lowman HB, Fischer SK. Evaluation of IgE antibodies to Omalizumab (Xolair ${ }^{\circledR}$ ) and their potential correlation to anaphylaxis. AAPS J. 18(1), 115-123 (2016).

69 Xue L, Clements-Egan A, Amaravadi L et al. Recommendations for the assessment and management of pre-existing drug-reactive antibodies during biotherapeutic development. AAPS J. 19(6), 1576-86 (2017).

70 Gorovits B, Wakshull E, Pillutla R, Xu Y, Manning MS, Goyal J. Recommendations for the characterization of immunogenicity response to multiple domain biotherapeutics. J. Immunol Methods 408, 1-12 (2014).

71 Manning MS, Kroenke MA, Lee SA et al. Assay signal as an alternative to titer for assessment of magnitude of an antidrug antibody response. Bioanalysis 9(23), 1849-1857 (2017).

72 Wadhwa M, Mytych DT, Bird C et al. Establishment of the first WHO erythropoietin antibody reference panel: report of an international collaborative study. J. Immunol. Methods 435, 32-42 (2016).

73 Mytych DT, Barger TE, King C et al. Development and characterization of a human antibody reference panel against erythropoietin suitable for the standardization of ESA immunogenicity testing. J. Immunol. Methods 382(1-2), 129-141 (2012).

74 Workshop on Immunogenicity Assessment of Biotechnology-Derived Therapeutic Proteins Workshop Summary. EMA, Human Medicines Evaluation Division, London, UK (2016).

75 Knezevic I, Griffiths E. WHO standards for biotherapeutics, including biosimilars: an example of the evaluation of complex biological products. Ann. NY Acad. Sci. 1407(1), 5-16 (2017).

76 Shankar G, Devanarayan V, Amaravadi L et al. Recommendations for the validation of immunoassays used for detection of host antibodies against biotechnology products. J. Pharm. Biomed. Anal. 48(5), 1267-1281 (2008).

77 Devanarayan V, Smith WC, Brunelle RL, Seger ME, Krug K, Bowsher RR. Recommendations for systematic statistical computation of immunogenicity cut points. AAPS J. 19(5), 1487-1498 (2017).

78 Kubiak R, Zhang L, Zhang J et al. Correlation of screening and confirmatory results in tiered immunogenicity testing by solution-phase bridging assays. J. Pharm. Biomed. Anal. 74, 235-45 (2013).

79 Muire-Sluis AR, Barrett YC, Devanarayan V et al. Recommendations for the design and optimization of immunoassays used in the detection of host antibodies against biotechnology products. J. Immunol. Methods 289(1-2), 1-16 (2004).

80 Dias D, Van Doren J, Schlottmann S et al. Optimization and validation of a multiplexed luminex assay to quantify antibodies to neutralizing epitopes on human papillomaviruses 6, 11, 16, and 18. Clin. Diagn. Lab Immunol. 12(8), 959-969 (2005).

81 Kumar SC, DelCarpini JA, Qu Q, Kane M, Gorovits B. Mitigation of pre-existing antibodies to a biotherapeutic in non-clinical species when establishing anti-drug antibody assay cutpoint. AAPS J. 19(1), 313-319 (2017). 
82 LeBlond D, Singer R, Xu L, Zeng R. Bayesian estimation and comparison of immunogenicity screening assay floating cut-point factors from pre-study and in-study results. J. Immunol. Methods 418, 84-100 (2015).

83 Barnett D, Granger V, Whitby L, Storie I, Reilly JT. Absolute CD4+ T-lymphocyte and CD34 ${ }^{+}$stem cell counts by single-platform flow cytometry: the way forward. Br. J. Haematol. 106(4), 1059-1062 (1999).

84 O'Gorman, Maurice RG, Nicholson JKA. Adoption of single-platform technologies for enumeration of absolute T-lymphocyte subsets in peripheral blood. Clin. Diagn Lab Immunol. 7(3), 333-335 (2000).

85 United States Pharmacopoiea. General Chapter $<1106.1>$ immunogenicity assays design and validation of assays to detect anti-drug neutralizing antibody. Pharmacopeial Forum (PF) 40(3), (2014).

\section{Authors}

An Song ${ }^{10}$, Priya Sriraman ${ }^{38}$, Ludovicus Staelens ${ }^{39}$, Giane Sumner ${ }^{4}$, Yongliang Sun ${ }^{3}$, Martin Ullmann ${ }^{40}$, Daniela Verthelyi ${ }^{14}$, Meenu Wadhwa ${ }^{18}$, Yow-Ming Wang ${ }^{14}$, Yuanxin $\mathrm{Xu}^{41}$, Haoheng Yan ${ }^{14}$, Tong-yuan Yang $^{8}$ \& Rong Zeng ${ }^{42}$

\section{Affiliations}

${ }^{1}$ Biogen, Cambridge, MA, USA

${ }^{2}$ Sanofi, Framingham, MA, USA

${ }^{3}$ Bristol-Myers Squibb, Princeton, NJ, USA

${ }^{4}$ Regeneron Pharmaceuticals, Tarrytown, NY, USA

${ }^{5}$ Pfizer, Andover, MA, USA

${ }^{6}$ AbbVie, Redwood City, CA, USA

${ }^{7}$ Caprion Biosciences, Montreal, QC, Canada

${ }^{8}$ Janssen R\&D, Spring House, PA, USA

${ }^{9}$ Syneos Health, Princeton, NJ, USA

${ }^{10}$ Genentech, South San Francisco, CA, USA

${ }^{11}$ Roche Pharma Research \& Early Development, Roche Innovation Center, Munich, Germany

${ }^{12}$ Tesaro, Waltham, MA, USA

${ }^{13}$ Shire, Lexington, MA, USA

${ }^{14}$ US FDA, Silver Spring, MD, USA

${ }^{15}$ Pfizer, San Diego, CA, USA

${ }^{16}$ Pfizer, Groton, CT, USA

${ }^{17}$ Health Canada, Ottawa, ON, Canada

${ }^{18}$ UK MHRA-NIBSC, London, UK

${ }^{19}$ Angelini Pharma, Pomezia, RM, Italy

${ }^{20}$ GlaxoSmithKline, King of Prussia, PA, USA

${ }^{21}$ AstraZeneca, Molndal, Sweden

${ }^{22}$ UK MHRA, London, UK

\author{
${ }^{23}$ Japan MHLW-NIHS, Kanagawa, Japan \\ ${ }^{24}$ Celerion, Lincoln, NE, USA \\ ${ }^{25}$ Merck \& Co., Inc., Kenilworth, NJ, USA \\ ${ }^{26}$ PPD, Richmond, VA, USA \\ ${ }^{27}$ Roche Pharma Research \& Early Development, Roche Innovation \\ Center Basel, Switzerland \\ ${ }^{28}$ Medlmmune, Gaithersburg, MD, USA \\ ${ }^{29}$ EMD Serono/Merck KGaA, Billerica, MA, USA \\ ${ }^{30}$ Finland Fimea, Helsinki, Finland \\ ${ }^{31}$ Medlmmune, South San Francisco, CA, USA \\ ${ }^{32}$ Teva Pharmaceuticals, West Chester, PA, USA \\ ${ }^{33}$ Neoteric, Princeton, NJ, USA \\ ${ }^{34}$ BDS Immunoassay Services, Tampa, FL, USA \\ ${ }^{35}$ CFABS, Laval, QC, Canada \\ ${ }^{36}$ BioMarin, Novato, CA, USA \\ ${ }^{37}$ Amgen, Thousand Oaks, CA, USA \\ ${ }^{38}$ Celgene, Summit, NJ, USA \\ ${ }^{39}$ UCB Biopharma, Braine L'Alleud, Belgium \\ ${ }^{40}$ Fresenius-Kabi, Aubonne, Switzerland \\ ${ }^{41}$ Alnylam Pharmaceuticals, Cambridge, MA, USA \\ ${ }^{42}$ Amador Bioscience, Pleasanton, CA, USA (formerly of OncoMed, \\ Redwood City, CA, USA)
}

Regulatory agencies disclaimer: The views expressed in this article are those of the authors and do not reflect official policy of the US FDA, Europe EMA, France ANSM, UK MHRA, Austria AGES, Brazil ANVISA, Health Canada, Japan MHLW, and Finland Fimea. No official endorsement by the FDA, EMA, ANSM, Health Canada, MHRA, AGES, ANVISA, MHLW or Fimea is intended or should be inferred. 
